\title{
ALMA resolves molecular clouds in metal-poor Magellanic Bridge $A^{\star}$
}

\author{
M. T. Valdivia-Mena ${ }^{1}$, M. Rubio ${ }^{1}$, A. D. Bolatto ${ }^{2}$, H. P. Saldaño ${ }^{3}$, and C. Verdugo ${ }^{4}$ \\ ${ }^{1}$ Departamento de Astronomía, Universidad de Chile, Santiago, Chile \\ e-mail: maria.valdivia@ug.uchile.cl \\ ${ }^{2}$ University of Maryland, MD, USA \\ ${ }^{3}$ Observatorio Astronómico de Córdoba, UNC, Argentina \\ ${ }^{4}$ Joint Alma Observatory (JAO), Alonso de Córdova 3107, Vitacura, Santiago, Chile
}

Received 2 December 2019 / Accepted 22 June 2020

\begin{abstract}
Context. The Magellanic Bridge is a tidal feature located between the Magellanic Clouds, containing young stars formed in situ. Its proximity allows high-resolution studies of molecular gas, dust, and star formation in a tidal low-metallicity environment.

Aims. Our goal is to characterize gas and dust emission in Magellanic Bridge A, the source with the highest $870 \mu \mathrm{m}$ excess of emission found in single-dish surveys.

Methods. Using the ALMA telescope including the Morita Array, we mapped a 3' field of view centered on the Magellanic Bridge A molecular cloud, in $1.3 \mathrm{~mm}$ continuum emission and ${ }^{12} \mathrm{CO}(2-1)$ line emission at subparsec resolution. This region was also mapped in continuum at $870 \mu \mathrm{m}$ and in ${ }^{12} \mathrm{CO}(2-1)$ line emission at $\sim 6 \mathrm{pc}$ resolution with the APEX telescope. To study its dust properties, we also use archival Herschel and Spitzer data. We combine the ALMA and APEX ${ }^{12} \mathrm{CO}(2-1)$ line cubes to study the molecular gas emission.

Results. Magellanic Bridge A breaks up into two distinct molecular clouds in dust and ${ }^{12} \mathrm{CO}(2-1)$ emission, which we call North and South. Dust emission in the North source, according to our best parameters from fitting the far-infrared fluxes, is $\approx 3 \mathrm{~K}$ colder than in the South source in correspondence to its less developed star formation. Both dust sources present large submillimeter excesses in LABOCA data: according to our best fits the excess over the modified blackbody (MBB) fit to the Spitzer/Herschel continuum is $E(870 \mu \mathrm{m}) \sim 7$ and $E(870 \mu \mathrm{m}) \sim 3$ for the North and South sources, respectively. Nonetheless, we do not detect the corresponding $1.3 \mathrm{~mm}$ continuum with ALMA. Our limits are compatible with the extrapolation of the MBB fits, and therefore we cannot independently confirm the excess at this longer wavelength. The ${ }^{12} \mathrm{CO}(2-1)$ emission is concentrated in two parsec-sized clouds with virial masses of around 400 and $700 M_{\odot}$. Their bulk volume densities are $n\left(\mathrm{H}_{2}\right) \sim 0.7-2.6 \times 10^{3} \mathrm{~cm}^{-3}$, higher than typical bulk densities of Galactic molecular clouds. The ${ }^{12} \mathrm{CO}$ luminosity to $\mathrm{H}_{2}$ mass conversion factor $\alpha_{C O}$ is 6.5 and $15.3 M_{\odot}\left(\mathrm{K} \mathrm{km} \mathrm{s}^{-1} \mathrm{pc}^{2}\right)^{-1}$ for the North and South clouds, calculated using their respective virial masses and ${ }^{12} \mathrm{CO}(2-1)$ luminosities. Gas mass estimates from our MBB fits to dust emission yields masses $M \sim 1.3 \times 10^{3} M_{\odot}$ and $2.9 \times 10^{3} M_{\odot}$ for North and South, respectively, a factor of $\sim 4$ higher than the virial masses we infer from ${ }^{12} \mathrm{CO}$.
\end{abstract}

Key words. ISM: clouds - Magellanic Clouds - ISM: molecules - submillimeter: ISM

\section{Introduction}

The Magellanic Bridge was first described through neutral hydrogen ( $\mathrm{H} \mathrm{I})$ observations as a gaseous bridge joining the Large Magellanic Cloud (LMC) and Small Magellanic Cloud (SMC; Hindman et al. 1963). It is a filamentary structure with lumps, holes, and shells, with an extent of 15 to $21 \mathrm{kpc}$, a total gas mass of $\sim 1.5 \times 10^{8} M_{\odot}$, and an $\mathrm{H}$ I column density between $10^{20}$ and $10^{21} \mathrm{~cm}^{-2}$ (Staveley-Smith et al. 1998; Muller et al. 2003a). Simulations suggest that the Magellanic Bridge is the result of a close gravitational interaction between the LMC and SMC that happened around 200-300 Myrs ago (Murai \& Fujimoto 1980; Gardiner et al. 1994; Besla et al. 2012). Star formation has and is taking place in the Magellanic Bridge: there are young stars and clusters of less than $100 \mathrm{Myr}$ in age (Bica \& Schmitt 1995; Harris 2007; Bica et al. 2015; Kalari et al. 2018) and evidence of current star formation in the form of $\mathrm{H} \alpha$ filamentary shells

\footnotetext{
* Reduced maps and images are only available at the CDS via anonymous ftp to cdsarc.u-strasbg.fr $(130.79 .128 .5)$ or via http:
} //cdsarc.u-strasbg.fr/viz-bin/cat/J/A+A/641/A97
(Muller \& Parker 2007) and young stellar objects (YSOs; Chen et al. 2014). Its metallicity is low and seems to present a gradient, from values similar to the SMC main body $\left(Z \sim 1 / 5 Z_{\odot}\right.$, Lee et al. 2005) to about half (or even less) of the SMC metallicity $\left(Z \sim 1 / 10 Z_{\odot}\right.$, Rolleston et al. 2003; Lehner et al. 2008). At a distance of almost $60 \mathrm{kpc}$, using the SMC as reference (Harries et al. 2003; Cioni et al. 2000), the Magellanic Bridge is the closest low-metallicity tidally influenced region outside of a dwarf galaxy; it allows detailed studies of star formation under unique physical conditions.

Stars form in molecular clouds, which are composed of molecular hydrogen gas $\left(\mathrm{H}_{2}\right)$ and dust. Studying the molecular component of low-metallicity galaxies helps to understand the process of star formation in metal-poor environments, like those present in high-redshift galaxies. To this end, the molecular component of the Magellanic Bridge has been studied through carbon monoxide (CO) and dust emission, both tracers of $\mathrm{H}_{2}$ gas, but these emissions are weaker than in the SMC main body (Meixner et al. 2013; Muller et al. 2014), and are therefore harder to detect. 


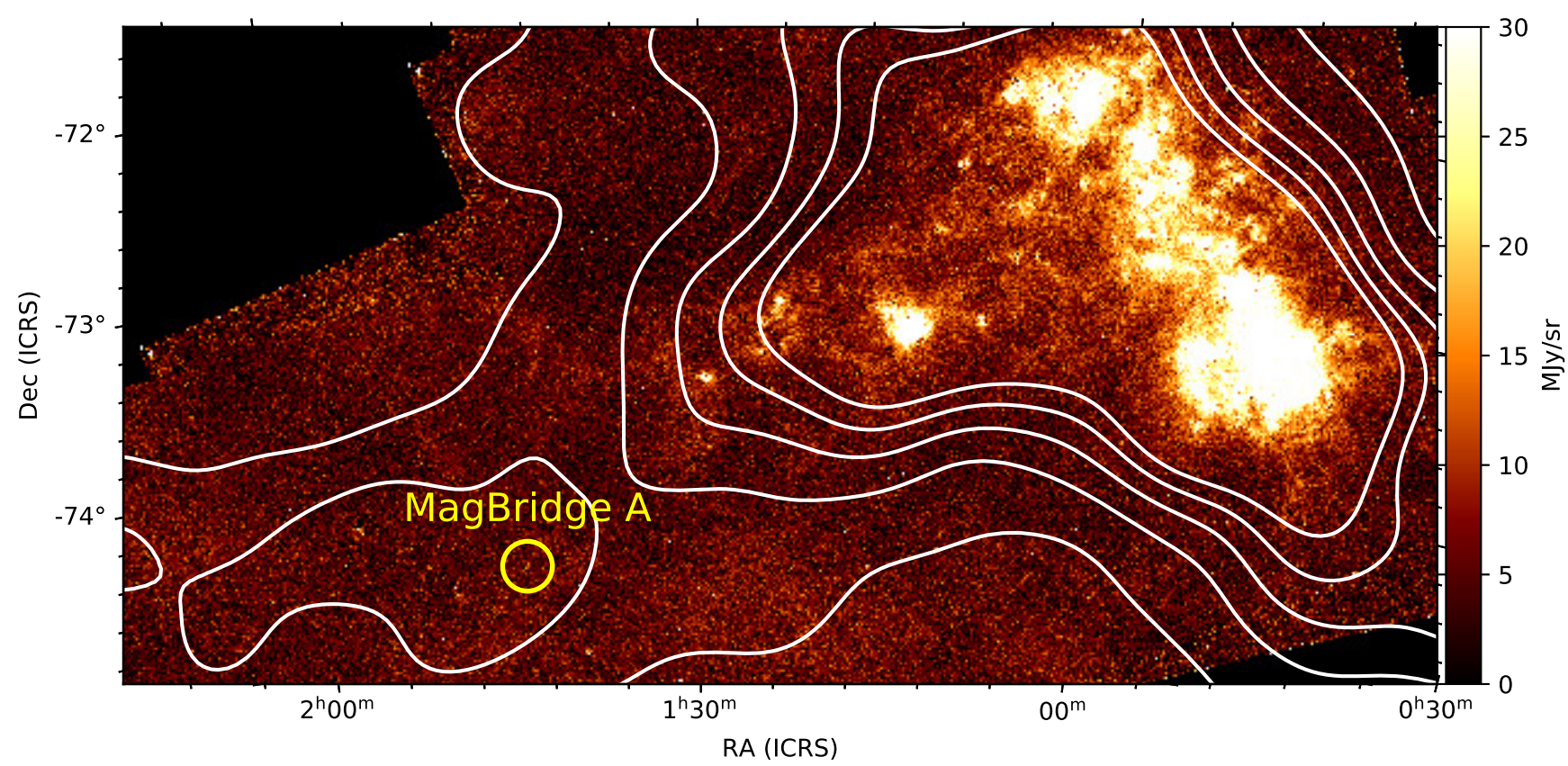

Fig. 1. Herschel $160 \mu \mathrm{m}$ map of the SMC from Meixner et al. (2013). White contours represent the H I column density map constructed using the Parkes Galactic All-Sky Survey (GASS) first data release (McClure-Griffiths et al. 2009). The contour levels are $(1,1.5,2,2.5$, and 3$) \times 10^{21} \mathrm{~cm}^{-2}$. The yellow circular region gives the position of Magellanic Bridge A (labeled MagBridge A).

The first study that detected a molecular cloud in the Magellanic Bridge was done by Muller et al. (2003b), using ${ }^{12} \mathrm{CO}(1-0)$ observations. The detection was found in a region with an $\mathrm{H}$ I intensity peak and where the 60 -to- $100 \mu \mathrm{m}$ intensity ratio $S_{60 \mu \mathrm{m}} / S_{100 \mu \mathrm{m}}<0.2$. Using a different criteria, matching H I and $100 \mu \mathrm{m}$ intensity peaks, Mizuno et al. (2006) detected seven more ${ }^{12} \mathrm{CO}$ clouds which, together with the Muller et al. (2003b) detected cloud, have ${ }^{12} \mathrm{CO}(1-0)$ intensities between 30 and $140 \mathrm{mK} \mathrm{km} \mathrm{s}^{-1}$ in a 2'.6 beam. These molecular clouds have narrow velocity widths $\left(\Delta v \lesssim 2 \mathrm{~km} \mathrm{~s}^{-1}\right)$, similar to the clouds in the metal-poor far edges of our galaxy, and molecular masses derived from their CO luminosities between $(1-7) \times 10^{3} M_{\odot}$ (Mizuno et al. 2006), similar to the masses of nearby molecular clouds in the Milky Way (Mizuno et al. 1995, 2001). The eight clouds have been labeled from $\mathrm{A}$ to $\mathrm{H}$, which is the naming convention that we use in this work. Mizuno et al. (2006) concluded that these clouds are a recent product of the Magellanic Bridge ISM and not remnants from the SMC. All of the clouds except for one are within a few tens of parsecs to the closest OB stellar association (Bica \& Schmitt 1995). YSO candidates have been found towards all of these sources, except for cloud D (Chen et al. 2014), showing the correlation of these molecular clouds with local star formation.

Dust, which is heated by star radiation and emits continuum at far-infrared (FIR) and millimeter wavelengths, also traces $\mathrm{H}_{2}$ gas. Observations in the FIR and millimeter regime have been done in the part of the Magellanic Bridge that is closest to the SMC, a section usually referred to as the SMC Tail (Gordon et al. 2011; Meixner et al. 2013). These observations reveal that dust in the Magellanic Bridge has a lower brightness than the SMC dust and a gas-to-dust ratio of $\sim 1200$, similar to the SMC gas-to-dust ratio (Gordon et al. 2011). Gordon et al. (2009) determined that dust in the Mizuno et al. (2006) molecular clouds B and C have temperatures of around 16-17 $\mathrm{K}$ and a gas-to-dust ratio lower than the rest of the Bridge, between 250 and 440.

The focus of our work is Magellanic Bridge source A, the molecular cloud that is closest to the SMC $(\sim 3.5 \mathrm{kpc}$ from the main body of the SMC; see Fig. 1). Magellanic Bridge A is one of the faintest of the eight molecular clouds detected by Mizuno et al. (2006), with a ${ }^{12} \mathrm{CO}(1-0)$ intensity of $30 \mathrm{mK} \mathrm{km} \mathrm{s}^{-1}$ and a molecular mass of $10^{3} M_{\odot}$, estimated from its ${ }^{12} \mathrm{CO}(1-0)$ emission. Magellanic Bridge A is located between two faint compact H II regions (Muller \& Parker 2007) and is within a stellar association (Bica \& Schmitt 1995). There is recent star formation in this region, revealed by two YSO candidates within the molecular cloud (Chen et al. 2014) and its proximity to H II regions and $24 \mu \mathrm{m}$ bright sources (Muller et al. 2014). Verdugo (2012) found that Magellanic Bridge A has very high excess emission at $870 \mu \mathrm{m}$ in comparison with other dust sources in the LMC and SMC.

The goal of this work is to characterize the gas and dust present in the Magellanic Bridge A molecular cloud at subparsec resolution. The Atacama Large Millimeter/Submillimeter Array (ALMA) telescope has the power to resolve gas and dust emission at the desired scales. With this characterization we can obtain the molecular gas properties in a region that is similar to low-metallicity galaxies at high redshift. In addition, we investigate the dust component of Magellanic Bridge A to characterize the submillimeter excess detected in this region (Verdugo 2012). To achieve our goal, we use high-resolution ALMA observations of Magellanic Bridge A together with observations from the Atacama Pathfinder Explorer (APEX) telescope, which reveal molecular gas and dust emission.

This article is organized as follows. In Sect. 2 we present new ALMA B6 continuum and ${ }^{12} \mathrm{CO}(2-1)$ line observations and complementary APEX observations, together with details of their reduction and imaging. In Sect. 3 we describe the submillimeter and millimeter emission and characterize the dust emission through its spectral energy distribution. In Sect. 4 we describe the molecular gas emission using ${ }^{12} \mathrm{CO}(2-1)$ and determine the physical properties of the detected ${ }^{12} \mathrm{CO}(2-1)$ sources. In Sect. 5 we discuss the results obtained for molecular gas and dust and compare them with previous observations of the ISM in Magellanic Bridge A. We summarize our results in Sect. 6. 


\section{Observations and data reduction}

In the following section, we describe the observations used to study the millimeter and submillimeter emission from Magellanic Bridge A. We summarize the main properties of the observations and describe the reduction process for the new data presented in this work.

\subsection{ALMA observations}

Magellanic Bridge A was observed with ALMA, located at Llano de Chajnantor in Atacama, Chile. We used Band 6 observations taken with the $12 \mathrm{~m}$ array and the $7 \mathrm{~m}$ array (also known as the Morita Array), which include continuum emission observations and the ${ }^{12} \mathrm{CO}(2-1)$ line emission data. In this paper we refer to the ${ }^{12} \mathrm{CO}(2-1)$ line as $\mathrm{CO}(2-1)$. Observations were performed in separate cycles: we used $7 \mathrm{~m}$ array data taken during Cycle 1 and $12 \mathrm{~m}$ array observations taken in Cycle 2. The datasets belong to project 2012.1.00683.S (PI: M. Rubio). As the observations were obtained and reduced in different cycles, we decided to start from the raw data and completely reprocess both datasets, instead of using the delivered data products by ALMA.

The $12 \mathrm{~m}$ observations were constructed with 33 antennas and consist of a mosaic of 93 pointings, with 13".7 spacing, and a total excecution time of $107 \mathrm{~min}$. Uranus was used as flux calibrator, J0057-7040 as phase calibrator, and J2357-5311 as bandpass calibrator.

The $7 \mathrm{~m}$ observations were conducted with ten antennas and consist of a mosaic of 34 pointings, with a distance of 23". 4 between the center of each pointing, and a total integration time of $93.7 \mathrm{~min}$. Uranus was also used as a flux calibrator for these observations. The phase calibrator was J0102-7546 and the bandpass calibrator was J2357-5311.

We reduced, combined, and imaged the data using the Common Astronomy Software Applications (CASA, McMullin et al. 2007) package version 4.7, with the consideration that the software was released later than the observations. We used the standard scripts provided by the ALMA Science Archive ${ }^{1}$ with the delivered raw data to recover the calibrated datasets. The $12 \mathrm{~m}$ and $7 \mathrm{~m}$ data were reduced separately.

We combined the calibrated $12 \mathrm{~m}$ and $7 \mathrm{~m}$ observations into one dataset. The observations were done using both the $12 \mathrm{~m}$ and $7 \mathrm{~m}$ array to have high-resolution data without losing extended emission: the $12 \mathrm{~m}$ array configuration gives us observations at high resolution, yet it filters out diffuse emission on a scale that exceeds the maximum recoverable scale (MRS), which for our $12 \mathrm{~m}$ observations is $18^{\prime \prime} 3$. The MRS increases to 30.9 after the $7 \mathrm{~m}$ array data are combined with the $12 \mathrm{~m}$ array observations. To combine data taken with two different arrays of antennas, it is crucial that the relative weights of each visibility are correct, as specified in the CASA guide ${ }^{2}$. We calculated the visibility weights of the $12 \mathrm{~m}$ and $7 \mathrm{~m}$ observations with the statwt task. After calculating the correct visibility weights, the $12 \mathrm{~m}$ array and $7 \mathrm{~m}$ array data were combined using the concat task with a frequency tolerance of $10 \mathrm{MHz}$ (using the freqtol parameter).

We imaged the combined $12 \mathrm{~m}$ and $7 \mathrm{~m}$ data and generated a continuum emission image and a $\mathrm{CO}(2-1)$ line cube. We describe the imaging process for the continuum map in Sect. 2.1.1 and the procedure to generate the $\mathrm{CO}(2-1)$ line cube in Sect. 2.1.2. We also combined the ALMA $\mathrm{CO}(2-1) 12 \mathrm{~m}$

\footnotetext{
1 http://almascience.eso.org//aq/

2 https://casaguides.nrao.edu/index.php/

DataWeightsAndCombination
}

Table 1. Properties of the resulting continuum at $1.3 \mathrm{~mm}(230 \mathrm{GHz})$.

\begin{tabular}{lllll}
\hline \hline Weight & $\begin{array}{l}\theta_{\text {major }} \\
\left({ }^{\prime \prime}\right)\end{array}$ & $\begin{array}{l}\theta_{\text {minor }} \\
\left({ }^{\prime \prime}\right)\end{array}$ & $\begin{array}{l}\text { PA } \\
\left.{ }^{\circ}\right)\end{array}$ & $\begin{array}{l}\sigma \\
\left(\mathrm{mJy} \mathrm{beam}^{-1}\right)\end{array}$ \\
\hline Natural & 1.54 & 1.37 & 47.9 & 0.17 \\
Natural+UVT (5") & 5.93 & 4.71 & -34.3 & 0.35 \\
Natural+UVT $\left(5^{\prime \prime}\right)^{(*)}$ & 22.0 & 22.0 & 0 & 1.20 \\
Briggs $(r=0.5)$ & 1.26 & 1.16 & 44.7 & 0.18 \\
\hline
\end{tabular}

Notes. UVT: uv-tapered images, using the tapering size in parentheses. ${ }^{(*)}$ Convolved to reach a beam size of $22^{\prime \prime}$.

and $7 \mathrm{~m}$ data with single-dish $\mathrm{CO}(2-1)$ observations obtained with the APEX telescope to include the zero spacing emission. The combination of ALMA and APEX data is described in Sect. 2.3.

\subsection{1. $1.3 \mathrm{~mm}$ continuum}

We used the standard clean task on the $12 \mathrm{~m}$ and $7 \mathrm{~m}$ combined data to generate the $1.3 \mathrm{~mm}(230 \mathrm{GHz})$ continuum image, removing the spectral channels that contain the $\mathrm{CO}(2-1)$ line. We generate the image using both natural weight and Briggs weight. The natural weight lowers the noise and allows point sources to be detected more effectively, but degrades the angular resolution; on the other hand, Briggs weighting (also known as robust weighting) provides better resolution and smaller sidelobes of the beam, with a penalty in noise. The Briggs weighted image is generated with a robust parameter $r=0.5$. The Briggs and natural weighted images resulted in nearly identical rms, $0.18 \mathrm{mJy} \mathrm{beam}^{-1}$ for Briggs weight and $0.17 \mathrm{mJy} \mathrm{beam}^{-1}$ for natural weight. We decided to use natural weight for this work as sensitivity tends to be higher for natural weighted images than Briggs weighted ones. The natural weighted continuum image is in Fig. 2. The continuum image has a field of view (FOV) of $3^{\prime} \times 3^{\prime}$.

The continuum image we use for this work is generated using natural weight and a tapering in the $u-v$ plane. The taper gives more weight to the shorter baselines, which might improve the sensitivity to extended sources but degrades the resolution of the image. We applied a tapering of $5^{\prime \prime}$, which results in an image with a resolution of $5 . \prime 9 \times 4{ }^{\prime \prime} 7$. To compare with the APEX LABOCA $870 \mu \mathrm{m}$ image (see Sect. 2.2.1), we convolved the natural, uv-tapered image to reach a beam FWHM equal to $22^{\prime \prime}$. The beam size (described through the FWHM in the major and minor axes $\theta_{\text {major }}$ and $\theta_{\text {minor }}$ ) and rms $\sigma$ of the continuum images, before and after tapering and convolution, are summarized in Table 1. The tapered continuum images (convolved and not convolved to $\left.22^{\prime \prime}\right)$ are in Fig. 2.

\subsection{2. $\mathrm{CO}(2-1)$ line}

We performed the clean standard routine in the combined $12 \mathrm{~m}$ and $7 \mathrm{~m}$ data, using a mask based on the APEX $\mathrm{CO}(2-1)$ data described in Sect. 2.2.2. We used Briggs weighting with a robust parameter of 0.5 . The reduced $\mathrm{CO}(2-1)$ cube has a FOV of $3^{\prime} \times 3^{\prime}$, a velocity resolution of $0.5 \mathrm{~km} \mathrm{~s}^{-1}$, an angular resolution of $1^{\prime \prime} .22 \times 11^{\prime \prime} 15\left(\mathrm{PA}=42^{\circ}\right)$, and variable rms throughout the line cube with values ranging from 22 and 36 mJy beam $^{-1}$ per channel.

This ALMA CO(2-1) cube was combined with the $\mathrm{CO}(2-1)$ APEX line cube as described in Sect. 2.3. 

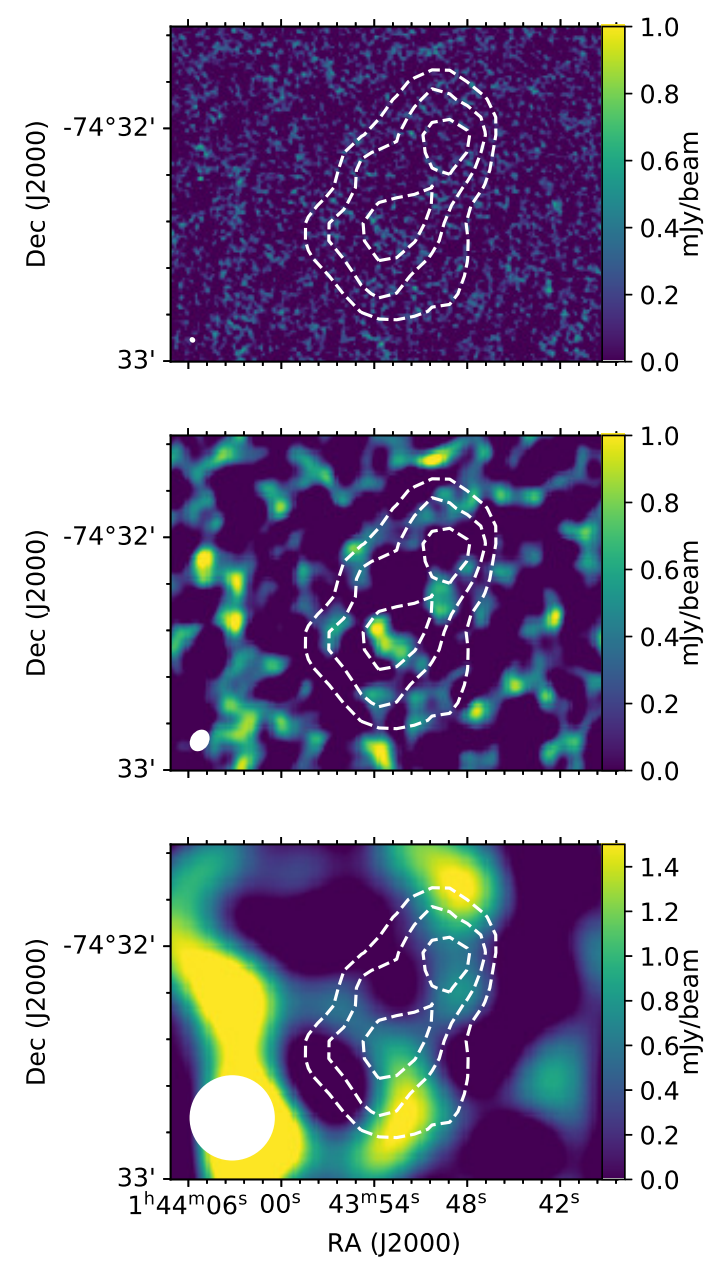

Fig. 2. ALMA continuum images at $1.3 \mathrm{~mm}$ of Magellanic Bridge A, generated using $12 \mathrm{~m}$ and $7 \mathrm{~m}$ data, where imaging is performed using natural weight. Top image: continuum without tapering in the visibility data. Middle image: continuum with its visibility data tapered using a Gaussian with an on-sky FWHM of 5". Bottom image: tapered continuum image convolved to reach a resolution of $22^{\prime \prime}$. The color scale for this last image is different from the other two images. The white ellipse in the bottom left corner of each image represents the beam size. Dashed contours correspond to continuum emission in the APEX $870 \mu \mathrm{m}$ map at 25,30 , and $35 \mathrm{mJy}^{\text {beam }}{ }^{-1}$.

\subsection{APEX observations}

We used observations of Magellanic Bridge A performed with the APEX telescope ${ }^{3}$, a $12 \mathrm{~m}$ diameter telescope located in Llano de Chajnantor, Chile. These observations consist of an $870 \mu \mathrm{m}$ continuum image, which we describe in Sect. 2.2.1, and a $\mathrm{CO}(2-1)$ line emission cube, described in Sect. 2.2.2.

\subsection{1. $0.87 \mathrm{~mm}$ continuum}

We used $870 \mu \mathrm{m}$ continuum observations of Magellanic Bridge A obtained with the Large APEX Bolometer Camera (LABOCA) on the APEX telescope. LABOCA is an array of bolometers with a central frequency of $345 \mathrm{GHz}$, a bandwidth of $60 \mathrm{GHz}$, and a total FOV of 11'.4 (Siringo et al. 2009).

\footnotetext{
3 APEX is a collaboration between the Max-Planck-Institut fur Radioastronomie, the European Southern Observatory, and the Onsala Space Observatory.
}

We used the $870 \mu \mathrm{m}$ continuum image of Magellanic Bridge A presented in Verdugo (2012). The Magellanic Bridge A data belongs to project C-086.F-0679A-2010 (PI: M. Rubio). The observations were done in August and October 2010, with a precipitable water vapor (pwv) content between 0.2 and $0.9 \mathrm{~mm}$. The total integration time of the LABOCA observations was $3.94 \mathrm{~h}$. The reduction of this continuum image was done with the Bolometric Array Analysis Software $(\mathrm{BoA})^{4}$. The reduction required two calibrations: an opacity $(\tau)$ calibration, using the $\tau$ values obtained from pwv measurements done with the APEX radiometer, and a flux calibration based on Neptune and Uranus flux observations. The flux calibration has an estimated error of $20 \%$. The final continuum image was generated through an iterative reduction process:

- first reduction, with the standard BoA reduction script optimized for weak sources;

- two iterations of the reduction code, flagging pixels with $\mathrm{S} / \mathrm{N}$ over 2.5 to get rid of extra noise;

- six repetitions of the reduction code using a mask to detect emission with $\mathrm{S} / \mathrm{N}$ higher than 2.5, subtracting the emission, reducing the map without the source, and adding the source again before the beginning of the next iteration.

The final image is presented in the bottom right box of Fig. 3 . It has a beam size of $22^{\prime \prime} .4(6.4 \mathrm{pc})$, a FOV of $11.4 \times 11 ! 4$, and an rms of $5 \mathrm{mJy}$ beam $^{-1}$, which is consistent with the integration time and weather conditions at the time of observation.

\subsection{2. $\mathrm{CO}(2-1)$ line}

We use $\mathrm{CO}(2-1)$ line observations, performed using the onthe-fly mapping technique with the APEX-1 receiver of the Swedish Heterodyne Facility Instrument (SHeFI) at the APEX telescope. The receiver has a spectral range of $213-275 \mathrm{GHz}$. These observations were made in June 2014, under project C093.F-9711A-2014 (PI: M. Rubio), with pwv between 1.0 and $1.6 \mathrm{~mm}$. The total integration time towards Magellanic Bridge A was $1.28 \mathrm{~h}$. The mapped area consists of a square of $5{ }^{\prime \prime} 9 \times 55^{\prime \prime} 9$ covering the central region of the infrared emission detected by the Herschel telescope (see Fig. 3).

We reduced the APEX CO(2-1) line cube through the standard procedure of the CLASS software, Gildas 5 . The antenna temperature $T_{A}^{*}$ is delivered by APEX corrected for atmospheric attenuation ${ }^{6}$. We scaled $T_{A}^{*}$ to the main beam brightness temperature using $T_{\mathrm{mb}}=T_{A}^{*} / \eta_{\mathrm{mb}}$, with a main beam efficiency $\eta_{\mathrm{mb}}=0.72$ for APEX-1 (Vassilev et al. 2008). The resulting APEX CO(2-1) cube has a spatial resolution of $28 . ' 7$, a velocity channel spacing of $0.125 \mathrm{~km} \mathrm{~s}^{-1}$, and an $\mathrm{rms}$ of $5.4 \mathrm{Jy}^{\text {beam }}{ }^{-1}$ $(150 \mathrm{mK})$ per channel. This line cube was combined with the resulting ALMA CO(2-1) line cube and described in Sect. 2.3.

\subsection{Combined ALMA-APEX CO(2-1) observations}

We combined the $12 \mathrm{~m}$ and $7 \mathrm{~m}$ ALMA $\mathrm{CO}(2-1)$ line cube described in Sect. 2.1.2 with the single-dish APEX $\operatorname{CO}(2-1)$ line cube from Sect. 2.2.2. To combine the observations we used the feather task from the CASA software, which performs the combination in Fourier space. The final combined

\footnotetext{
$4 \mathrm{BoA}$ is a data reduction package developed specially to process data acquired with bolometer arrays at APEX telescope. The primary goal is to handle data observed with LABOCA.

5 http://www.iram. fr/IRAMFR/GILDAS/

6 http://www.apex-telescope.org/observing/ (APECS user manual, Revision 4.1 June 5, 2020).
} 

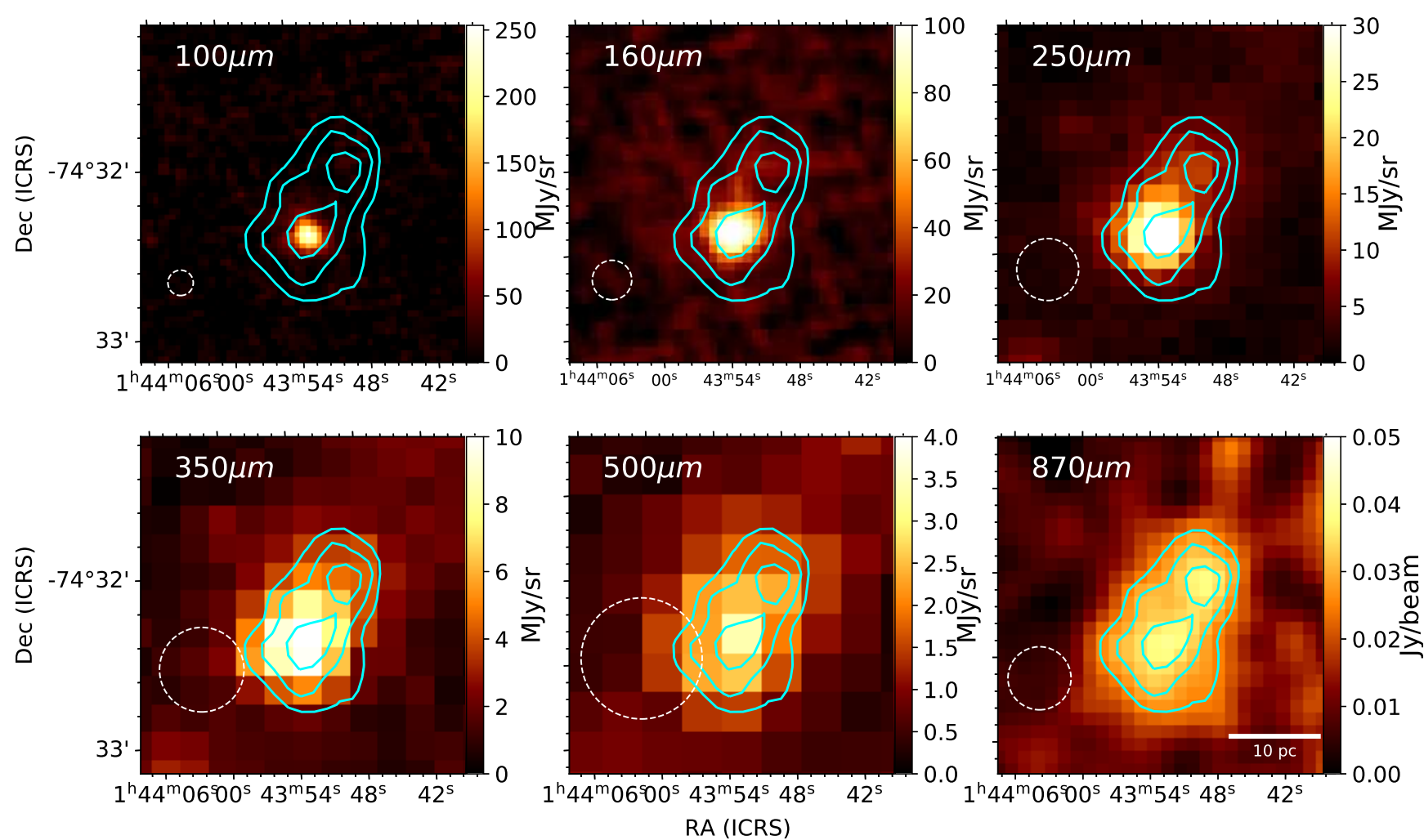

Fig. 3. Herschel continuum images of Magellanic Bridge A at 100, 160, 250, 350, and $500 \mu \mathrm{m}$, together with the LABOCA continuum image at $870 \mu \mathrm{m}$. The cyan contours correspond to the LABOCA $870 \mu \mathrm{m}$ continuum image at $25(5 \sigma), 30(6 \sigma)$, and $35 \mathrm{mJy}^{2} \mathrm{beam}^{-1}(7 \sigma)$. The dashed circles indicate the beam sizes for each image, which correspond to a FWHM of 9" $(100 \mu \mathrm{m}), 14^{\prime \prime}(160 \mu \mathrm{m}), 22^{\prime \prime}(250 \mu \mathrm{m}), 30^{\prime \prime}(350 \mu \mathrm{m}), 43^{\prime \prime}(500 \mu \mathrm{m})$, and 22.'4 $(870 \mu \mathrm{m})$. The scale bar in the bottom right panel indicates a $10 \mathrm{pc}$ length.

$\mathrm{CO}(2-1)$ datacube has a FOV of $3^{\prime} \times 3^{\prime}$, a spatial resolution of $1^{\prime \prime} .22 \times 1^{\prime \prime} .15(\approx 0.3 \mathrm{pc})$ and spectral resolution of $0.5 \mathrm{~km} \mathrm{~s}^{-1}$. This $\mathrm{CO}(2-1)$ line cube has a variable rms, with values between 30 and $36 \mathrm{mJy}^{\text {beam }}{ }^{-1}$ per channel depending on position.

\subsection{Complementary data}

We used Herschel observations from the HERITAGE Herschel key project (Meixner et al. 2013), consisting of maps of the SMC at $100,160,250,350$, and $500 \mu \mathrm{m}$. We extract a square region of $11^{\prime} \times 11^{\prime}$ from these maps, centered at $\alpha=1^{\mathrm{h}} 43^{\mathrm{m}} 50^{\mathrm{s}}, \delta=$ $-74^{\circ} 32^{\prime} 23^{\prime \prime}$ (FK5, J2000) to work with the continuum images centered at Magellanic Bridge A. The angular resolution of the $100,160,250,350$, and $500 \mu \mathrm{m}$ images is $9^{\prime \prime}, 14^{\prime \prime}, 22^{\prime \prime}, 30^{\prime \prime}$, and $43^{\prime \prime}$ and the rms noise of each image is $9,6,0.6,0.3$, and $0.2 \mathrm{MJy} \mathrm{sr}^{-1}$, respectively. The flux calibration of these images has an associated uncertainty of 10 and $20 \%$ for the 100 and $160 \mu \mathrm{m}$ images, respectively, and $\sim 8 \%$ for the 250,350 , and $500 \mu \mathrm{m}$ images. We also use the $160 \mu \mathrm{m}$ Spitzer image obtained from the Multiband Imaging Photometer for Spitzer (MIPS) for the SAGE-SMC Spitzer Legacy Program (Gordon et al. 2011), and extracted the region containing Magellanic Bridge $\mathrm{A}$ in the same way. The Spitzer image has a resolution of $40^{\prime \prime}$, an rms of $4 \mathrm{MJy} \mathrm{sr}^{-1}$, and flux calibration uncertainty of $\sim 10 \%$. All these images were taken from the NASA/IPAC Infrared Science Archive ${ }^{7}$.

We used an $\mathrm{H} \alpha$ image of the SMC from the Southern $\mathrm{H} \alpha$ Sky Survey Atlas (SHASSA; Gaustad et al. 2001), which contains the

https://irsa.ipac.caltech.edu/frontpage/
Magellanic Bridge. The survey covers $13^{\circ}$ of the southern sky and has a resolution of about 0.8 . We extract the region containing the Magellanic Bridge A source in the same way as with the Hershel images.

\section{Millimeter and submillimeter emission from Magellanic Bridge A}

In this section we study the Magellanic Bridge A dust emission through the millimeter and submillimeter continuum images described in Sects. 2.1.1, 2.2.1, and 2.4. We first describe the sources present in the $870 \mu \mathrm{m}$ continuum image. Then we present the spectral energy distribution (SED) of Magellanic Bridge A dust we constructed using the continuum images and subtracting all contributions that do not come from dust emission (Sect. 3.1). We obtained the dust properties of Magellanic Bridge A by adjusting a Modified Blackbody (MBB) model to the SED (Sect. 3.2). We compare the resulting MBB model with the dust emission derived from the LABOCA $870 \mu \mathrm{m}$ and ALMA $1.3 \mathrm{~mm}$ continuum images (Sect. 3.3). Finally, we used the properties obtained from the model to calculate the total gas mass using dust emission (Sect. 3.4).

We present three different ALMA $1.3 \mathrm{~mm}$ continuum images using different weights and tapered as explained in Sect. 2.1.1; no continuum source was detected in these ALMA continuum images, as seen in Fig. 2. There is a hint of emission in the uvtapered ALMA image convolved to $22^{\prime \prime}$, but this emission is not coincident with Magellanic Bridge A in the continuum images from Herschel, Spitzer, or LABOCA, so it might be related to antenna noise generating some artifacts. We used the natural 
Table 2. Dust emission flux densities in Magellanic Bridge A North and South.

\begin{tabular}{llllllll}
\hline \hline Source & $\begin{array}{l}R_{\mathrm{ap}} \\
\left({ }^{\prime \prime}\right)\end{array}$ & $\begin{array}{l}S_{100 \mu \mathrm{m}} \\
(\mathrm{mJy})\end{array}$ & $\begin{array}{l}S_{160 \mu \mathrm{m}} \\
(\mathrm{mJy})\end{array}$ & $\begin{array}{l}S_{250 \mu \mathrm{m}} \\
(\mathrm{mJy})\end{array}$ & $\begin{array}{l}S_{350 \mu \mathrm{m}} \\
(\mathrm{mJy})\end{array}$ & $\begin{array}{l}S_{500 \mu \mathrm{m}} \\
(\mathrm{mJy})\end{array}$ & $\begin{array}{l}S_{870 \mu \mathrm{m}}^{(\dagger)} \\
(\mathrm{mJy})\end{array}$ \\
\hline MagB. A $^{(*)}$ & 50 & $952 \pm 95 \pm 151$ & $1565 \pm 313 \pm 511$ & $856 \pm 68 \pm 48$ & $422 \pm 34 \pm 33$ & $\begin{array}{l}S_{1.3 \mathrm{~mm}}^{(\ddagger)} \\
(\mathrm{mJy})\end{array}$ \\
North $^{(* *)}$ & 11 & $131 \pm 13 \pm 49$ & $305 \pm 61 \pm 120$ & $139 \pm 11 \pm 12$ & $\ldots$ & $\ldots$ & $257 \pm 52 \pm 28<16$ \\
South $^{(* *)}$ & 11 & $678 \pm 68 \pm 48$ & $838 \pm 168 \pm 120$ & $449 \pm 36 \pm 13$ & $\ldots$ & $\ldots$ & $46 \pm 9 \pm 5$ \\
\hline
\end{tabular}

Notes. We express the errors on our measurements with the flux calibration error first (Herschel errors obtained from Meixner et al. 2013) and the photometric error last. ${ }^{(\dagger)}$ Flux at $870 \mu \mathrm{m}$ has the free-free emission and $\mathrm{CO}(3-2)$ line contributions subtracted. ${ }^{(\ddagger)}$ Flux at $1.3 \mathrm{~mm}$ has the free-free emission contribution subtracted. ${ }^{(*)}$ In Magellanic Bridge A, all images are convolved to a common resolution of $43^{\prime \prime} .{ }^{(* *)}$ In the North and South sources all images are convolved to a common resolution of $22^{\prime \prime}$.

weighted uv-tapered image to obtain an upper limit for the flux density at $1.3 \mathrm{~mm}$ (see Sect. 3.1).

We detected continuum emission from Magellanic Bridge A at $870 \mu \mathrm{m}$. The APEX continuum image is shown in the bottom right panel of Fig. 3. The source was also detected in the Herschel 100, 160, 250, 350, and $500 \mu$ m images. There are two peaks with similar intensities $\left(\sim 40 \mathrm{mJy} \mathrm{beam}^{-1}\right)$ and separated by $\sim 27^{\prime \prime}(8 \mathrm{pc})$, which is near the limit of the APEX resolution. We identify these two sources as North and South throughout this paper. In the Herschel 100 and $160 \mu \mathrm{m}$ images, the northern peak is not seen, while the southern source can be seen in all images. Unlike the APEX $870 \mu \mathrm{m}$ continuum, the 350 and $500 \mu \mathrm{m}$ lower resolution images do not separate well the northern component from the southern one, but it can be seen as an elongated structure. For comparison, we plot the APEX contours over the Herschel continuum images in Fig. 3. In the following section, we characterize the dust emission towards Magellanic Bridge $\mathrm{A}$, and we also characterize the emission coming from the North and South sources found in the APEX $870 \mu \mathrm{m}$ image.

\subsection{Spectral energy distribution of Magellanic Bridge A}

We built the SED for Magellanic Bridge A using Herschel, Spitzer, APEX, and ALMA continuum images. We also built the SED for the North and South sources using Herschel emissions only at 100,160 and $250 \mu \mathrm{m}$, together with the LABOCA and ALMA continuum images. To construct each SED, we performed aperture photometry at each wavelength where emission was detected to obtain the fluxes. Then, we subtracted the contributions that are not thermal emission. We determined an upper flux limit in the ALMA $1.3 \mathrm{~mm}$ continuum image, as no emission was detected.

In the following, we describe how we constructed the SED and how we determined the contribution from other emission mechanisms.

\subsubsection{Flux measurements}

We obtained the fluxes in the Herschel, Spitzer, and LABOCA continuum images of Magellanic Bridge A using aperture photometry. We first convolved all images to a common resolution of $43^{\prime \prime}$, which corresponds to the resolution of the Herschel $500 \mu \mathrm{m}$ image, as it has the poorest resolution of all our images. Convolving results in a change in the rms of each image: the new rms are 2.0, 5.0, 0.5, and 0.3 $\mathrm{MJy} \mathrm{sr}^{-1}$ for the Herschel 100, 160, 250, and $350 \mu \mathrm{m}$ images, respectively. The convolved LABOCA image has an rms of $26 \mathrm{mJy}_{\text {beam }}^{-1}$. We measured the total emission coming from Magellanic Bridge $\mathrm{A}$ in an aperture with a radius $r=50^{\prime \prime}$, centered at $\alpha=1^{\mathrm{h}} 43^{\mathrm{m}} 52^{\mathrm{s}} .68, \delta=-74^{\circ} 32^{\prime} 20^{\prime} .23$ (FK5,
J2000). For all images, we subtracted the sky using an annulus centered at the same position as the aperture, with inner radius $r_{\text {in }}=2.5$ and outer radius $r_{\text {out }}=3$ ' .0 for Magellanic Bridge A. We did not apply an aperture correction to the photometry results of Magellanic Bridge A because the aperture encloses the source completely and the aperture area is almost four times the beam size. The total flux error is the sum in quadrature of the flux calibration error for the measured flux and the photometric error $\epsilon$ :

$\epsilon=\sqrt{N} \sigma$.

Here $N$ is the number of beams inside the aperture and $\sigma$ is the rms of the image in $\mathrm{Jy}_{\text {beam }}{ }^{-1}$. The fluxes measured for Magellanic Bridge A are given in the first row of Table 2. The $870 \mu \mathrm{m}$ and $1.3 \mathrm{~mm}$ emission in this table have been corrected for free-free emission and $\mathrm{CO}(2-1)$ line contribution at the respective wavelength. These corrections are further explained in Sect. 3.1.2. In the Spitzer $160 \mu \mathrm{m}$ image (not included in Table 2 ), we measured a flux density $S_{160 \mu \mathrm{m}}=1009 \pm 101 \pm 386$, where the first error corresponds to the flux calibration error and the second is the photometric error.

In the case of Magellanic Bridge A North and South, we only used the 100,160 , and $250 \mu \mathrm{m}$ images from Herschel and the LABOCA $870 \mu \mathrm{m}$ continuum. We did not use the 350 and $500 \mu \mathrm{m}$ images because the North and South sources cannot be separated due to the resolution (their beam FWHM are larger than 22"). We convolved the Herschel images to a common resolution of $22^{\prime \prime}$ to measure their fluxes. The rms after convolution are 2.2 and $5.5 \mathrm{MJy} \mathrm{sr}^{-1}$ for the Herschel 100 and $160 \mu \mathrm{m}$ images, respectively. For the photometry, we used a circular aperture with a common radius $r=11^{\prime \prime}$, centered at $\alpha=1^{\mathrm{h}} 43^{\mathrm{m}} 49^{\mathrm{s}} .0, \delta=-74^{\circ} 32^{\prime} 00^{\prime} \cdot 2$ (FK5, J2000) for source North and at $\alpha=1^{\mathrm{h}} 43^{\mathrm{m}} 53.8, \delta=$ $-74^{\circ} 32^{\prime} 24^{\prime \prime} 5$ (FK5, J2000) for source South, which correspond to the peak positions in the LABOCA image. In this case, since the sources are not resolved and the photometry is performed within the FWHM of the spatial beam, we applied an aperture correction factor of 2 in the flux measurements. The total flux error was also calculated as the sum in quadrature of the associated flux calibration error and photometric error.

Since there is no emission detected in the ALMA $1.3 \mathrm{~mm}$ image, we determined an upper limit for the flux at $1.3 \mathrm{~mm}$ for the source Magellanic Bridge A and sources North and South, based on the ALMA continuum image sensitivity. To do this we used a value of three times the photometric error $\epsilon$. For Magellanic Bridge A we obtained a photometric error $\epsilon=5.5 \mathrm{mJy}$ inside an $r=50^{\prime \prime}$ circular aperture, which gives a $1.3 \mathrm{~mm}$ flux upper limit for Magellanic Bridge A of $16.5 \mathrm{mJy}$. The 

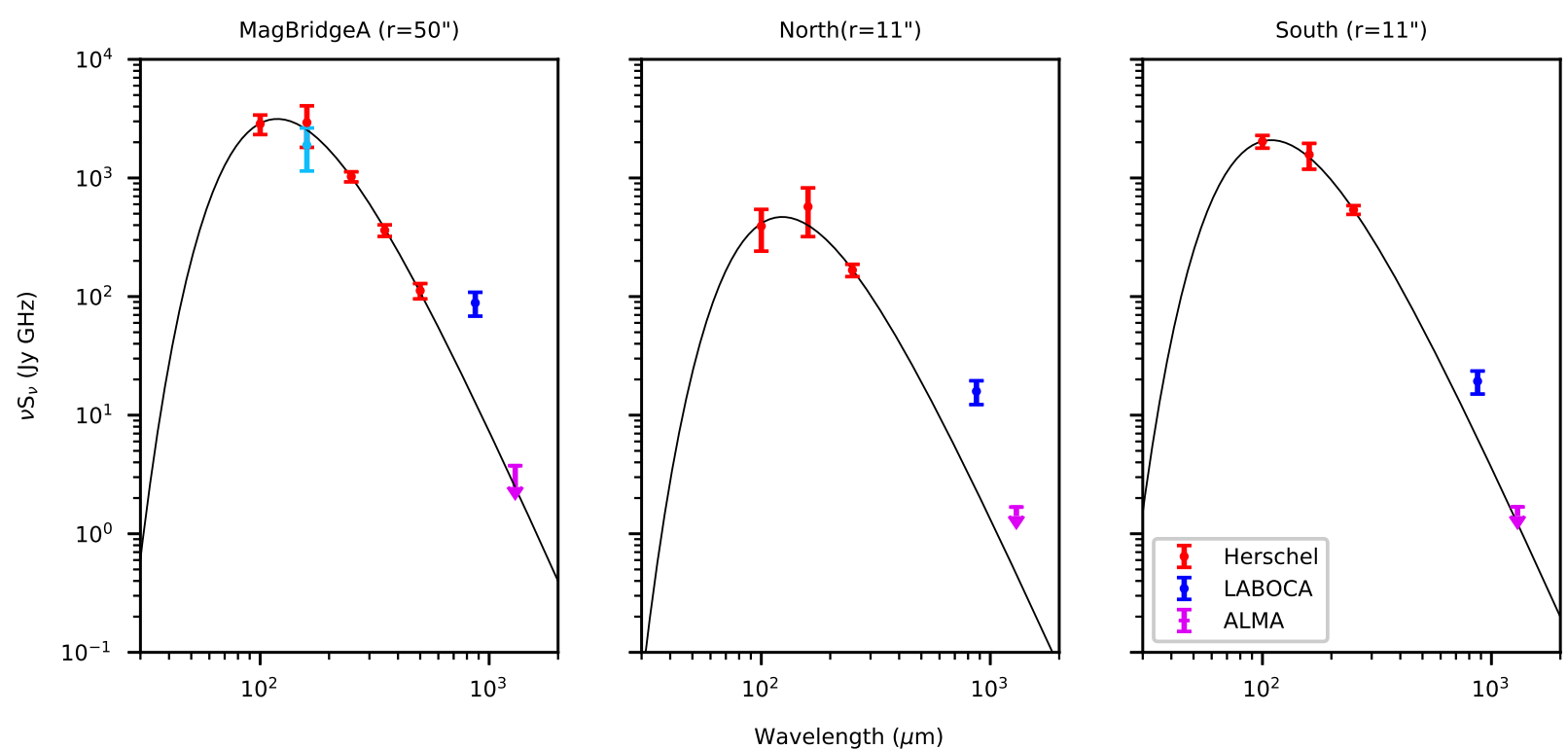

Fig. 4. SEDs for Magellanic Bridge A and the North and South sources. The points correspond to the energy $v S_{v}$ derived from the measured flux density $S_{\lambda}$, obtained using aperture photometry. Red points correspond to $v S_{v}$ obtained using Herschel data. Dark blue points correspond to the energy $v S_{v}$ obtained using the LABOCA image. The purple arrow corresponds to the $v S_{v}$ upper limit at $1.3 \mathrm{~mm}$ using the ALMA continuum image. Left panel: Magellanic Bridge A SED, for which were used the 100, 160, 250, 350, and $500 \mu \mathrm{m}$ Herschel continuum images; the $160 \mu \mathrm{m}$ Spitzer continuum image; and the $870 \mu \mathrm{m}$ (LABOCA) and $1.3 \mathrm{~mm}$ (ALMA) continuum images, all with a common resolution of $43^{\prime \prime}$.The flux from Magellanic Bridge A was measured inside a circular aperture of radius $r=50^{\prime \prime}$. The North and South sources SEDs are shown in the last two panels, constructed with the Hershel 100, 160, and $250 \mu$ m continuum images, the $870 \mu \mathrm{m}$ (LABOCA) and $1.3 \mathrm{~mm}$ (ALMA) continuum images at a common resolution of 22". The fluxes from sources North and South were measured in a circular aperture of radius $r=11^{\prime \prime}$. The MBB model that best fits the Herschel points for each source is shown as a black curve. The parameters of each curve are listed in Table 4 .

photometric error for the North and South sources is $\epsilon=2.4 \mathrm{mJy}$ in a $r=11^{\prime \prime}$ circular aperture, after applying an aperture correction of a factor of 2. This gives a $1.3 \mathrm{~mm}$ flux upper limit of $7.2 \mathrm{mJy}$ for sources North and South.

The fluxes measured for each source and wavelength, together with the flux upper limit at $1.3 \mathrm{~mm}$, are summarized in Table 2. The $1.3 \mathrm{~mm}$ and $870 \mu \mathrm{m}$ fluxes listed correspond to dust emission only at these wavelengths as free-free emission and $\mathrm{CO}$ line contributions have been estimated and subtracted (see Sect. 3.1.2). Using these fluxes, we built the SED shown in Fig. 4.

\subsubsection{Free-free and $\mathrm{CO}(3-2)$ contribution}

The flux measurement from the APEX $870 \mu \mathrm{m}$ image contains emission of three different physical processes: (1) thermal dust continuum emission, (2) free-free (bremsstrahlung) emission from ionized gas, and (3) a contribution from molecular lines in the passband $\left({ }^{12} \mathrm{CO}(3-2)\right.$ in particular, which is the brightest). At $1.3 \mathrm{~mm}$, there is also free-free emission and a molecular line $\left({ }^{12} \mathrm{CO}(2-1)\right)$. We are interested in the dust continuum emission; therefore, we need to calculate and remove the other two contributions to the measured flux. The $\mathrm{CO}(2-1)$ line emission was already removed when the ALMA $1.3 \mathrm{~mm}$ continuum image is constructed (see Sect. 2.1.1), so we determined the contribution of free-free to both $870 \mu \mathrm{m}$ and $1.3 \mathrm{~mm}$ continuum emission and the contribution of ${ }^{12} \mathrm{CO}(3-2)$ line emission in the $870 \mu \mathrm{m}$ continuum image.

We determined the free-free contribution to continuum emission using $\mathrm{H} \alpha$ emission as the intensity of the $\mathrm{H} \alpha$ line traces the warm ionized gas component of the ISM and, therefore, is proportional to the free-free emission (see the Appendix in Hunt et al. 2004). To convert $\mathrm{H} \alpha$ flux density into free-free emission
$S_{v}^{f f}$ in mJy, we used the following formula:

$S_{v}^{f f}=1.16\left(1+\frac{n\left(\mathrm{He}^{+}\right)}{n\left(\mathrm{H}^{+}\right)}\right)\left(\frac{T_{\mathrm{e}}}{10^{4}}\right)^{0.62} v^{-0.1}\left(\frac{S_{\alpha}}{10^{-12}}\right)$.

Here $S_{\alpha}$ is the intensity of $\mathrm{H} \alpha$ in erg $\mathrm{cm}^{-2} \mathrm{~s}^{-1} ; T_{\mathrm{e}}$ is the gas electron temperature in $\mathrm{K} ; n\left(\mathrm{He}^{+}\right)$and $n\left(\mathrm{H}^{+}\right)$are the number density of helium and hydrogen ions, respectively; and $v$ is the frequency where we need to determine the free-free emission in $\mathrm{GHz}$. To obtain the intensity of $\mathrm{H} \alpha$ emission in Magellanic Bridge A we used the $\mathrm{H} \alpha$ image from SHASSA (Gaustad et al. 2001), which is in decirayleighs $(\mathrm{dR})$. We first transformed the map units to rayleighs (R) dividing by 10 and then to erg $\mathrm{cm}^{-2} \mathrm{~s}^{-1} \mathrm{sr}^{-1}$ using $1 \mathrm{R}=h v \times 10^{6} /(4 \pi)=2.41 \times 10^{-7} \mathrm{erg} \mathrm{cm}^{-2} \mathrm{~s}^{-1} \mathrm{sr}^{-1}$, where $v$ is the frequency corresponding to the $\mathrm{H} \alpha$ line $\left(4.57 \times 10^{14} \mathrm{~Hz}\right)$. The $\mathrm{H} \alpha$ measurements obtained through aperture photometry in the same positions as in Sect. 3.1.1 are $(1.2 \pm 0.2) \times$ $10^{-13} \mathrm{erg} \mathrm{cm}^{-2} \mathrm{~s}^{-1}$ for Magellanic Bridge A, and $(2.3 \pm 0.3) \times$ $10^{-14} \mathrm{erg} \mathrm{cm}^{-2} \mathrm{~s}^{-1}$ and $(2.4 \pm 0.4) \times 10^{-14} \mathrm{erg} \mathrm{cm}^{-2} \mathrm{~s}^{-1}$ for the North and South sources, respectively. These values consider an extinction $A_{V}=0.140$, obtained for the position of Magellanic Bridge A (Schlafly \& Finkbeiner 2011). We used $T_{\mathrm{e}} \sim 1.7 \times 10^{4}$ $\mathrm{K}$, the upper limit for the gas temperature reported in Lehner et al. (2001), and $n\left(\mathrm{He}^{+}\right) / n\left(\mathrm{H}^{+}\right) \sim 0.08$, which is the value estimated for low-metallicity sources like the Magellanic Clouds (Hunt et al. 2004).

After applying Eq. (2) using $v=344.8 \mathrm{GHz}(870 \mu \mathrm{m})$, we obtained a free-free contribution of $117 \pm 22 \mu \mathrm{Jy}$ for Magellanic Bridge A, $22 \pm 4 \mu \mathrm{Jy}$ for cloud North, and $23 \pm 4 \mu \mathrm{Jy}$ for cloud South. These values represent $\lesssim 0.05 \%$ of the total continuum emission. When we used Eq. (2) with $v=230.8 \mathrm{GHz}(1.3 \mathrm{~mm})$, we obtained a free-free contribution of $122 \pm 23 \mu \mathrm{Jy}$ in Magellanic Bridge A, $23 \pm 4 \mu \mathrm{Jy}$ in cloud North and $24 \pm 4 \mu \mathrm{Jy}$ 
Table 3. Free-free emission for each source at $870 \mu \mathrm{m}$ and $1.3 \mathrm{~mm}$, obtained through aperture photometry.

\begin{tabular}{lll}
\hline \hline Source & $S_{0.87 \mathrm{~mm}}^{f f}(\mathrm{mJy})$ & $S_{1.3 \mathrm{~mm}}^{f f}(\mathrm{mJy})$ \\
\hline Magellanic Bridge $^{(*)}$ & $0.117 \pm 0.022$ & $0.122 \pm 0.023$ \\
North $^{(* *)}$ & $0.022 \pm 0.004$ & $0.023 \pm 0.004$ \\
South $^{(* *)}$ & $0.023 \pm 0.004$ & $0.024 \pm 0.004$ \\
\hline
\end{tabular}

Notes. ${ }^{(*)}$ Aperture photometry done in a circle with a radius of $50^{\prime \prime}$. ${ }^{(* *)}$ Aperture photometry done in a circle with a radius of $11^{\prime \prime}$.

in cloud South. The values for each source and wavelength are listed in Table 3 . The fluxes presented in Table 2 have these contributions subtracted.

We obtained the ${ }^{12} \mathrm{CO}(3-2)$ molecular line contribution to the LABOCA continuum flux $S_{\mathrm{CO}(3-2)}$ in Magellanic Bridge A using Eq. (5) of Drabek et al. (2012). We calculate the total flux density present in Magellanic Bridge A from the peak integrated intensity $I_{\mathrm{CO}(3-2)}$ using

$S_{{ }^{12} \mathrm{CO}(3-2)}=\frac{2 k v^{3}}{c^{3} \Delta v_{\mathrm{bol}}} \Omega I_{{ }^{12} \mathrm{CO}(3-2)}$,

where $I_{\mathrm{CO}(3-2)}$ is in $\mathrm{K} \mathrm{km} \mathrm{s}^{-1}, \Delta v_{\text {bol }}$ is the bandpass width in $\mathrm{Hz}, v$ is the frequency of the ${ }^{12} \mathrm{CO}(3-2)$ line in $\mathrm{Hz}, \Omega$ is the source area in sr, and $S_{\mathrm{CO}(3-2)}$ is in Jy. We approximated the LABOCA spectral response to a function that is constant over the LABOCA bandpass, so that $g(v)_{\text {line }} / \int g(v) \mathrm{d} v=1 / \Delta v_{\text {bol }}$. We used $I_{12} \mathrm{CO}(3-2)=0.93 \pm 0.07 \mathrm{~K} \mathrm{~km} \mathrm{~s}^{-1}$, the value for the peak $I_{12} \mathrm{CO}(3-2)$ in Magellanic Bridge A given in Table 2 of Muller et al. (2014), and its area $\Omega$ corresponds to one single ASTE pointing, with $F W H M=22^{\prime \prime}$. The resultant ${ }^{12} \mathrm{CO}(3-2)$ line contribution to the continuum emission at $870 \mu \mathrm{m}$ is $S_{\mathrm{CO}(3-2)}=0.85 \pm 0.06 \mathrm{mJy}$. This value is subtracted from the total flux in Magellanic Bridge A at 870 microns, but not from the North and South sources as there is only one source detected in ${ }^{12} \mathrm{CO}(3-2)$. Nevertheless, the estimated ${ }^{12} \mathrm{CO}(3-2)$ contribution is less than $2 \%$ of the $870 \mu \mathrm{m}$ flux in sources North and South.

We used the APEX $870 \mu$ m flux measurement with the freefree and ${ }^{12} \mathrm{CO}(3-2)$ line emission contribution subtracted, the ALMA $1.3 \mathrm{~mm}$ flux upper limit with free-free contribution subtracted, and the FIR flux densities obtained from Herschel and Spitzer images to construct the SED of Magellanic Bridge A and the North and South sources. The three SEDs are plotted in Fig. 4.

\subsection{SED modeling}

We used the SED constructed in Sect. 3.1.2 for Magellanic Bridge A to obtain the dust temperature and dust emissivity index of the region. We also used the SEDs of sources North and South to obtain their dust temperatures. Each SED was modeled assuming that the emission comes from an MBB, which consists of the Planck function multiplied by an emissivity that depends on frequency and dust properties. We derived the physical properties of these dust sources using the parameters of the MBB that best fit each source SED.

At millimeter and submillimeter wavelengths, we can assume that dust emission is optically thin, which means we can write the power per unit area, frequency, and solid angle as $I_{v}=\tau_{v} B_{v}\left(T_{d}\right)$, where $\tau_{v}$ is the dust optical depth and $B_{v}$ is the Planck's law value at the dust temperature $T_{d}$. The optical depth can be defined through the dust absorption coefficient (in $\mathrm{cm}^{2} \mathrm{~g}^{-1}$ ) as $\tau_{v}=\kappa_{d}(v) \Sigma_{d}$, where $\Sigma_{d}$ is the surface density of the source in $\mathrm{g} \mathrm{cm}^{-2}$. Replacing $\tau_{v}$ and integrating $I_{v}$ by the solid angle we obtain

$S_{v}=\Omega \kappa_{d}(v) \Sigma_{d} B_{v}\left(T_{d}\right)$,

where $\Omega$ is the solid angle of the source.

In the MBB model we write the absorption coefficient $\kappa_{d}(v)$ as a function of the spectral emissivity index $\beta$ as $\kappa_{d}(v)=\kappa_{d}\left(v_{0}\right)\left(v / v_{0}\right)^{\beta}$, where $\kappa_{d}\left(v_{0}\right)$ is the absorption coefficient at the reference frequency $v_{0}$. Replacing $\kappa_{d}(v)$ and reorganizing the constant values $\Omega, \Sigma_{d}, \kappa_{d}\left(v_{0}\right)$, and $v_{0}$ as $C$, the flux density is a function of frequency $v$, emissivity index $\beta$, and dust temperature $T_{d}$ (Hildebrand 1983):

$S_{v}=C v^{\beta} B_{v}\left(T_{d}\right)$

We fitted the MBB model described by Eq. (5) for Magellanic Bridge A using the 100, 160, 250, 350, and $500 \mu \mathrm{m}$ fluxes, obtained from Herschel images in a $r=50^{\prime \prime}$ radius aperture (see Sect. 3.1.1). We assumed a single dust component and single $\beta$ dust emissivity index. There are three free parameters in the model: $C, \beta$, and $T_{d}$. We used $\chi^{2}$ minimization to determine $C, \beta$, and $T_{d}$ that best fit the SED, using the function curve_fit of the scipy.optimize package (Virtanen et al. 2020). The MBB that best fits the SED of Magellanic Bridge A has a dust temperature $T_{d}=22.4 \pm 3.4 \mathrm{~K}$ and an emissivity index $\beta=1.4 \pm 0.5$, listed in Table 4. In Fig. 4, the solid black line in the left panel represents the best fit MBB for the SED of Magellanic Bridge A.

We fitted the MBB model for the North and South sources only using the 100,160 , and $250 \mu \mathrm{m}$ fluxes because the 350 and $500 \mu \mathrm{m}$ beams do not resolve two sources and have poorer resolution than $22^{\prime \prime}$ (the $870 \mu \mathrm{m}$ beam). For these sources, we assume that the dust emissivity is the same as that found for Magellanic Bridge A, and we therefore fixed $\beta=1.4$ for both sources. We note that assuming a $\beta$ value for these sources influences the dust temperature obtained, as there is an inverse correlation between $\beta$ and $T_{d}$ (Shetty et al. 2009). We found the parameters $C$ and $T_{d}$ that best fit the SEDs with $\chi^{2}$ minimization. The temperature obtained for source South is $T_{d}=24.5 .4 \pm 2.2 \mathrm{~K}$ and source North $T_{d}=21.7 .4 \pm 1.1 \mathrm{~K}$; these parameters are listed in Table 4, and the best fit curves are plotted in Fig. 4 (black line). The best fit results indicate that the North source is colder than the South source by $\sim 3 \mathrm{~K}$.

\subsection{Submillimeter excess}

We define the submillimeter and millimeter excesses $E(\lambda)$ as the ratio of the measured flux to the predicted flux from the SED model at $\lambda=870 \mu \mathrm{m}$ and $1.3 \mathrm{~mm}$. We calculate these excesses for each source and summarize our results in Table 5.

The submillimeter excess found at $870 \mu \mathrm{m}$ for Magellanic Bridge $A$ is $E(870 \mu \mathrm{m})=6.9 \pm 1.6$ inside an circular aperture with $r=50^{\prime \prime}$. When we separate Magellanic Bridge A into the North and South sources, this submillimeter excess is also present. For the North source, $E(870 \mu \mathrm{m})=6.7 \pm 1.6$, while in the South is $E(870 \mu \mathrm{m})=3.0 \pm 1.4$, both considering a photometry aperture radius $r=11^{\prime \prime}$. Excess emission at $870 \mu \mathrm{m}$ has been detected in other low-metallicity galaxies using LABOCA (Galametz et al. 2009, 2014; Hermelo et al. 2016) and SCUBA observations (Galliano et al. 2003, 2005; Bendo et al. 2006). 
Table 4. Gas masses and best fit MBB parameters to the cloud SEDs.

\begin{tabular}{llllll}
\hline \hline Source & $\mathrm{C}\left(\mathrm{sr} \mathrm{Hz}^{-\beta}\right)$ & $\beta$ & $T_{d}(\mathrm{~K})$ & $\chi^{2}$ & $M_{\text {gas }}\left(10^{3} M_{\odot}\right)^{(\ddagger)}$ \\
\hline MagBridgeA $^{(*)}$ & $(1.9 \pm 23.4) \times 10^{-29}$ & $1.4 \pm 0.5$ & $22.4 \pm 3.4$ & 0.27 & $6.8 \pm 1.5$ \\
North $^{(* *)}$ & $(9.0 \pm 3.1) \times 10^{-30}$ & $1.4^{(\dagger)}$ & $21.7 \pm 2.2$ & 0.5 & $1.3 \pm 0.5$ \\
South $^{(* *)}$ & $(2.1 \pm 0.4) \times 10^{-29}$ & $1.4^{(\dagger)}$ & $24.5 \pm 1.1$ & 0.04 & $2.9 \pm 0.5$ \\
\hline
\end{tabular}

Notes. ${ }^{(*)}$ Fluxes measured using aperture photometry radius of $50^{\prime \prime} .{ }^{(*)}$ Fluxes measured using aperture photometry radius of $11^{\prime \prime} .{ }^{(\dagger)} \beta$ values obtained for Magellanic Bridge A MBB fit. ${ }^{(\ddagger)}$ Gas masses derived from the MBB models that best fit the FIR SEDs of each source.

Table 5. Submillimeter and millimeter excesses for Magellanic Bridge A and sources North and South.

\begin{tabular}{lll}
\hline \hline Source & $E(870)$ & $E(1.3 \mathrm{~mm})$ \\
\hline MagBridgeA & $6.9 \pm 1.6$ & $<1.5$ \\
North & $6.7 \pm 1.6$ & $<3.7$ \\
South & $3.0 \pm 0.7$ & $<1.4$ \\
\hline
\end{tabular}

Notes. Excess is defined as the ratio of the observed flux density to the flux density predicted by the best fit MBB model to the FIR dust fluxes in the SED.

Since we do not detect our sources in the $1.3 \mathrm{~mm}$ ALMA continuum, we calculate how much higher the flux upper limits calculated in Sect. 3.1.1 are than the predicted flux densities at $1.3 \mathrm{~mm}$. We derive an expected flux using the MBB model that best fits the FIR SED (see Table 4). We obtain an expected flux density of $10.7 \mathrm{mJy}$ for Magellanic Bridge A in an aperture radius $r=50^{\prime \prime}$, and $2.0 \mathrm{mJy}$ for the North source and 5.3 mJy for the South source within an aperture of radius $r=11^{\prime \prime}$. The flux upper limit at $1.3 \mathrm{~mm}$ is $16.5 \mathrm{mJy}$, which is 1.5 times higher than the expected flux density using the MBB model fitted to the FIR fluxes for Magellanic Bridge A. For the individual North and South sources, the upper limits from the ALMA continuum image at $1.3 \mathrm{~mm}$ are $7.2 \mathrm{mJy}$ (3.6 times higher than the expected flux density for source North) and $2.2 \mathrm{mJy}$ (1.4 times higher for source South). These values are reported as $E(1.3 \mathrm{~mm})$ in Table 5. In summary, our ALMA $1.3 \mathrm{~mm}$ data are compatible, within the detection limit, with the MBB fit to the FIR observations, and show no excess at this wavelength. If the excess at 1.3 $\mathrm{mm}$ were similar to that at $870 \mu \mathrm{m}$, we would have detected the source.

The fact that we do not observe emission at $1.3 \mathrm{~mm}$ when there is notable excess emission at $870 \mu \mathrm{m}$ in our sources is puzzling. The calibration of the LABOCA data was verified as correct by members of our team and the instrument support team, who performed independent reductions of the data that resulted in very similar images. In addition, we note that the morphology of the source at $870 \mu \mathrm{m}$ in Fig. 3 is consistent with emission at 250 to $500 \mu \mathrm{m}$ continuum images, and the noise of the map is consistent with that expected for the integration time. We would have only detected the South source at $\sim 3 \sigma$ in the complex if there were no excess. Therefore, we have every expectation that the $870 \mu \mathrm{m}$ excess is real. If this is so, there are two possibilities to explain the lack of a $1.3 \mathrm{~mm}$ detection: either the anomalous emission at $870 \mu \mathrm{m}$ is present only at that wavelength or the dust emission is too extended and resolved out in the ALMA $1.3 \mathrm{~mm}$ observation. We note that it is very difficult to explain the $870 \mu \mathrm{m}$ excess as resulting from an additional very cold dust component, as the lack of an excess at $500 \mu \mathrm{m}$ would require it to have a dust temperature $T_{d} \sim 3 \mathrm{~K}$. Through the inclusion of the Morita Array our

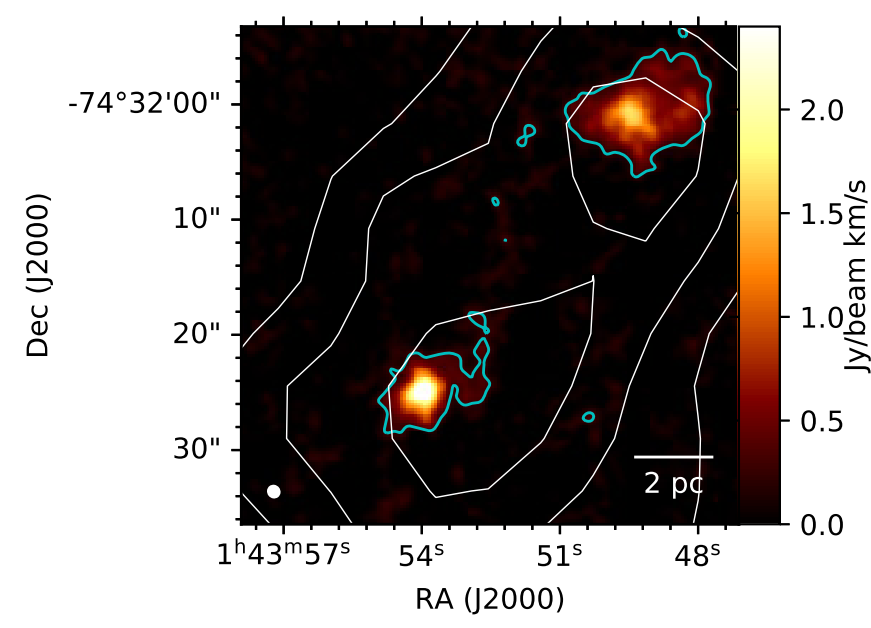

Fig. 5. Velocity integrated intensity image of the combined ALMA and APEX CO(2-1) cube of Magellanic Bridge A, integrated between 172 and $176.0 \mathrm{~km} \mathrm{~s}^{-1}$. The cyan contours correspond to 5 times the $\sigma$ of the integrated image $\left(\sigma=48 \mathrm{mJy}_{\text {beam }}{ }^{-1} \mathrm{~km} \mathrm{~s}^{-1}\right.$ ). The white contours correspond to the LABOCA $870 \mu \mathrm{m}$ continuum image at 25,30 , and $35 \mathrm{mJy}$ beam $^{-1}$, as in Fig. 3. The white ellipse in the lower left corner represents the beam FWHM $\left(1.22^{\prime \prime} \times 1.15^{\prime \prime}\right)$. The scale bar in the lower right corner indicates a $2 \mathrm{pc}$ length.

ALMA observations have a maximum recoverable scale (MRS) of $30.9^{\prime \prime}$, although our sensitivity substantially degrades close to that limit. We would expect to see continuum sources similar in size to the $\mathrm{CO}$ emitting clouds in this region (Fig. 5) if their $1.3 \mathrm{~mm}$ flux had an excess over the MBB fit similar to that measured at $870 \mu \mathrm{m}$. If the sources were larger than the $22^{\prime \prime}$ used for the photometry, however, they would be at least partially resolved out and undetectable. Clearly future ALMA observations determining the sizes of the sources at $870 \mu \mathrm{m}$ or deeper observations at $1.3 \mathrm{~mm}$ would be extremely useful to establish the nature of the excess.

\subsection{Gas masses obtained from dust emission}

We calculate the total gas masses associated with the $870 \mu \mathrm{m}$ dust emission for Magellanic Bridge A predicted by the MBB model that best fits the FIR fluxes. We use the same method to obtain the gas mass from dust flux used in Bot et al. (2010) and Rubio et al. (2004). The dust flux $S_{v}$ is related to the gas mass $M_{\text {gas }}$ through

$M_{\mathrm{gas}}=\frac{S_{v} D^{2} \mu \mathrm{m}_{\mathrm{H}}}{\epsilon_{d}(v) B_{v}\left(T_{d}\right)}$,

where $S_{v}$ is the predicted flux at frequency $v$ by the MBB model that best fits the FIR data in Jy; $D$ is the distance to the source in 
centimeters (we use the estimated distance to the SMC, $60 \mathrm{kpc}$; Harries et al. 2003); $\mu \mathrm{m}_{\mathrm{H}}$ is the gas weight per hydrogen atom in grams, including the contribution of helium ${ }^{8} ; \epsilon_{d}(v)$ is the emissivity of dust per hydrogen atom at frequency $v$ in $\mathrm{cm}^{2}$; and $B_{v}\left(T_{d}\right)$ is the spectral radiance at a dust temperature $T_{d}$ in $\mathrm{Jy}$ $\mathrm{sr}^{-1}$.

We use the $\epsilon_{d}(345 \mathrm{GHz})$ value presented in Bot et al. (2010), $\epsilon_{d}(345 \mathrm{GHz})=(3.94 \pm 0.05) \times 10^{-27} \mathrm{~cm}^{2}$, which assumes an absorption coefficient $\kappa_{345 \mathrm{GHz}}=1.26 \pm 0.02 \mathrm{~cm}^{2} \mathrm{~g}^{-1}$ and a dustto-gas ratio that is $1 / 6$ of the ratio in the solar neighborhood. This dust-to-gas ratio is similar to the ratios derived in Magellanic Bridge B and C (Gordon et al. 2009).

For Magellanic Bridge A source, North source, and South source, we use the dust temperatures $T_{d}=22.4 \pm 3.4 \mathrm{~K}$, $T_{d}=21.7 \pm 2.2 \mathrm{~K}$, and $T_{d}=24.5 \pm 1.1 \mathrm{~K}$, respectively, obtained from the best fit MBB to the Magellanic Bridge A SED with $\beta=1.4$, as derived in Sect. 3.2.

The total gas mass are calculated using the $870 \mu$ m flux predicted from the MBB fit. For Magellanic Bridge A, the gas mass is $(6.8 \pm 1.5) \times 10^{3} M_{\odot}$ and for the two separate clouds, North and South, their gas masses are $(1.3 \pm 0.5) \times 10^{3} M_{\odot}$ and $(2.9 \pm 0.5) \times 10^{3} M_{\odot}$, respectively. There is a difference between the sum of the gas masses of sources North and South and the gas mass of Magellanic Bridge A because dust measurements for the North and South sources are obtained in an aperture of radius $r=11^{\prime \prime}$, which only includes the peak emission of each cloud, while Magellanic Bridge A is measured inside an aperture of radius $r=50^{\prime \prime}$, which captures the complete emission from the source. We also estimated the gas masses using an absorption coefficient determined for dust emission at $160 \mu \mathrm{m}$ by Gordon et al. (2014), $\kappa_{160 \mu \mathrm{m}}=9.6 \pm 2.5 \mathrm{~cm}^{2} \mathrm{~g}^{-1}$, as the gas masses are dependant on the chosen $\epsilon_{d}(v)$, which depends on the calibration of $\kappa_{\gamma}$. Using $\kappa_{160 \mu \mathrm{m}}$ and the same gas-to-dust ratio (1/6 of the value in the solar neighborhood), we obtain a gas mass for Magellanic Bridge A of $(9.6 \pm 4.4) \times 10^{3} M_{\odot}$, while for source North it is $(1.7 \pm 0.8) \times 10^{3} M_{\odot}$ and for source South $(3.9 \pm 1.4) \times 10^{3} M_{\odot}$. These values are within the uncertainties of those obtained using the Bot et al. (2010) value. For the rest of this work, we use the total gas masses derived from the Bot et al. (2010) calibration, listed in the last column of Table 4.

\section{Resolved CO(2-1) molecular clouds}

In this section, we study the $\mathrm{CO}(2-1)$ molecular line emission in Magellanic Bridge A. We produced the velocity integrated intensity image between 172 and $176 \mathrm{~km} \mathrm{~s}^{-1}$ of the ALMA and APEX CO(2-1) line cube (see Fig. 5). We measured an rms of $48 \mathrm{mJy}$ beam ${ }^{-1} \mathrm{~km} \mathrm{~s}^{-1}$ in this image, a value that is consistent with the calculated values of 42 and $50 \mathrm{mJy}_{\text {beam }}^{-1} \mathrm{~km} \mathrm{~s}^{-1}$ using the rms range of the velocity interval (see Sect. 2.3). We resolved two parsec-sized $\mathrm{CO}(2-1)$ clouds, which are located towards the North and South $870 \mu \mathrm{m}$ continuum sources. We extracted the spectra of each of these molecular clouds (Sect. 4.1) and determined the physical properties of the molecular clouds in this region (Sect. 4.2).

\section{1. $\mathrm{CO}(2-1)$ molecular clouds spectra}

We identified the molecular clouds in the ALMA and APEX $\mathrm{CO}(2-1)$ line cube and extracted their spectra. We first identified potential sources in the velocity integrated intensity image as all emission greater than $3 \sigma$, being $\sigma$ the rms of the integrated

\footnotetext{
$8 \mu=1.36$.
}

image. We define the area of each source as the number of pixels inside the $3 \sigma$ contour. Then we measured the second moments of emission along the major and minor axes of each source in the $\mathrm{CO}(2-1)$ integrated emission image. We only kept those sources whose minor second moment is larger than the beam size. The only sources that met this criterion (survived this decimation) are the two brightest $\mathrm{CO}(2-1)$ sources seen in Fig. 5, which we call $\mathrm{CO}$ clouds from now on. These $\mathrm{CO}$ clouds spatially coincide with the dust sources characterized in Sect. 3, as seen in Fig. 5. The peak $I_{\mathrm{CO}(2-1)}$ of these clumps in the $\mathrm{CO}(2-1)$ integrated image are $1.67 \pm 0.05 \mathrm{Jy}_{\text {beam }}^{-1} \mathrm{~km} \mathrm{~s}^{-1}$ in the North $\mathrm{CO}$ cloud and $2.81 \pm 0.05 \mathrm{Jy}_{\text {beam }}^{-1} \mathrm{~km} \mathrm{~s}^{-1}$ in the South CO cloud.

We obtained the integrated velocity spectra of each source, adding all spectra in the source area. The resulting spectra have an rms noise of $\sigma=0.30 \mathrm{Jy} \mathrm{km} \mathrm{s}^{-1}$ for $\mathrm{CO}$ cloud North and $\sigma=0.25 \mathrm{Jy} \mathrm{km} \mathrm{s}^{-1}$ for CO cloud South, in a velocity channel width $0.5 \mathrm{~km} \mathrm{~s}^{-1}$. We fitted a Gaussian profile to the velocity integrated spectra of clouds North and South to obtain the central velocity $v_{\mathrm{LSR}}$ in $\mathrm{km} \mathrm{s}^{-1}$, velocity dispersion $\sigma_{v}$ in $\mathrm{km} \mathrm{s}^{-1}$ and integrated flux density $S_{\mathrm{CO}(2-1)} \Delta v$ in $\mathrm{Jy} \mathrm{km} \mathrm{s}^{-1}$. We adjusted the Gaussian model to the spectra through least-squares fitting. We corrected $\sigma_{v}$ for broadening due to the finite spectral resolution, using Eq. (10) of Rosolowsky \& Leroy (2006) to obtain the deconvolved velocity dispersion $\sigma_{v \text {,dc }}$, and report the velocity FWHM $\Delta v$ calculated from $\sigma_{v \text {,dc }}$. We did not extrapolate $\sigma_{v}$ to correct for a sensitivity bias (as stated in Rosolowsky \& Leroy 2006), as the peak emission of the CO clouds North and South is detected with a $\mathrm{S} / \mathrm{N}$ of 33 and 56 , respectively.

The CO cloud North is centered at a velocity of $174.6 \pm 0.6 \mathrm{~km} \mathrm{~s}^{-1}$ with a velocity FWHM of $1.33 \pm$ $0.03 \mathrm{~km} \mathrm{~s}^{-1}$, and the CO cloud South is centered at $174.0 \pm 0.9 \mathrm{~km} \mathrm{~s}^{-1}$ with a FWHM of $1.93 \pm 0.05 \mathrm{~km} \mathrm{~s}^{-1}$. The clouds are centered at similar velocities and have similar velocity linewidths. The CO North cloud shows a peak $I_{\mathrm{CO}(2-1)}$ emission of $1.67 \pm 0.05 \mathrm{Jy} \mathrm{beam}^{-1} \mathrm{~km} \mathrm{~s}^{-1}$ and cloud South has a peak $I_{\mathrm{CO}(2-1)}$ emission of $2.81 \pm 0.05 \mathrm{Jy} \mathrm{beam}^{-1} \mathrm{~km} \mathrm{~s}^{-1}$. However, as CO cloud North covers an area of $8.1 \mathrm{pc}^{2}$, while $\mathrm{CO}$ cloud South covers $5.8 \mathrm{pc}^{2}$, the North cloud has a larger emission than the South, with a total flux density $S_{\mathrm{CO}(2-1)} \Delta v$ of $30.67 \pm 2.86 \mathrm{Jy} \mathrm{km} \mathrm{s}^{-1}$ and $21.65 \pm 2.05 \mathrm{Jy} \mathrm{m} \mathrm{s}^{-1}$, respectively.

The results of these fits are summarized in Table 6 .

\subsection{Physical properties}

We derived the sizes, $\mathrm{CO}(2-1)$ luminosities, and virial masses for the $\mathrm{CO}(2-1)$ clouds we found in Magellanic Bridge A using the parameters obtained from the integrated spectra for each cloud.

We calculated the radius using the second moments of emission, as suggested in Sect. 2.1 of Rosolowsky \& Leroy (2006) using $R=1.91 \sigma_{r} D$, where $\sigma_{r}$ is the geometric mean of the spatial second moments of emission in radians, corrected for the spatial broadening due to the beam as indicated by their Eq. (9), and $D$ is the distance to the cloud in pc. We assumed that Magellanic Bridge $\mathrm{A}$ is at a distance of $\sim 60 \mathrm{kpc}$, the same as the distance to the SMC (Harries et al. 2003). We did not extrapolate the radius $\sigma_{r}$ as we do not expect a significant change in the size $\sigma_{r}$, for the same reason as we did not do it for $\sigma_{v}$ : both CO sources were detected with high $\mathrm{S} / \mathrm{N}$ values as can be seen in Fig. 5. The radius obtained for the CO clouds are $1.29 \pm 0.07 \mathrm{pc}$ for cloud North and $1.04 \pm 0.04$ pc for cloud South.

We calculated the $\mathrm{CO}(2-1)$ luminosities in $\mathrm{K} \mathrm{km} \mathrm{s}^{-1} \mathrm{pc}^{2}$ using $L_{\mathrm{CO}(2-1)}=611.5 S_{\mathrm{CO}(2-1)} \Delta v D^{2}$, where $S_{\mathrm{CO}(2-1)} \Delta \mathrm{v}$ is the 
Table 6. Characteristics of the clouds identified in the $\mathrm{CO}(2-1)$ line cube.

\begin{tabular}{llllllll}
\hline \hline CO Source & $\begin{array}{l}\text { RA } \\
(\mathrm{J} 2000)\end{array}$ & $\begin{array}{l}\text { Dec } \\
(\mathrm{J} 2000)\end{array}$ & $\begin{array}{l}A \\
\left(\mathrm{pc}^{2}\right)\end{array}$ & $\begin{array}{l}\text { Peak I } I_{\mathrm{CO}(2-1)}^{(a)} \\
\left(\mathrm{Jy} \mathrm{beam}^{-1} \mathrm{~km} \mathrm{~s}^{-1}\right)\end{array}$ & $\begin{array}{l}v_{\mathrm{LSR}}{ }^{(b)} \\
\left(\mathrm{km} \mathrm{s}^{-1}\right)\end{array}$ & $\begin{array}{l}\Delta v(F W H M)^{(b)} \\
\left(\mathrm{km} \mathrm{s}^{-1}\right)\end{array}$ & $\begin{array}{l}S_{\mathrm{CO}(2-1)} \Delta v^{(b)} \\
\left(\mathrm{Jy} \mathrm{km} \mathrm{s}^{-1}\right)\end{array}$ \\
\hline North & $1: 43: 49.2$ & $-74: 32: 00.6$ & 8.1 & $1.67 \pm 0.05$ & $174.6 \pm 0.6$ & $1.33 \pm 0.03$ & $30.67 \pm 2.86$ \\
South & $1: 43: 53.8$ & $-74: 32: 24.7$ & 5.8 & $2.81 \pm 0.05$ & $174.0 \pm 0.9$ & $1.93 \pm 0.05$ & $21.65 \pm 2.05$ \\
\hline
\end{tabular}

Notes. ${ }^{(a)}$ Peak values obtained from the $\mathrm{CO}(2-1)$ integrated line image between 172 and $176 \mathrm{~km} \mathrm{~s}^{-1}$. ${ }^{(b)}$ Values obtained from the Gaussian fit to the integrated spectra over the cloud area.

Table 7. Physical properties of the emission found in the ${ }^{12} \mathrm{CO}(2-1)$ line cube.

\begin{tabular}{lllll}
\hline \hline CO source & $\begin{array}{l}R^{(*)} \\
(\mathrm{pc})\end{array}$ & $\begin{array}{l}M_{\text {vir }} \\
\left(M_{\odot}\right)\end{array}$ & $\begin{array}{l}L_{\mathrm{CO}(2-1)} \\
\left(\mathrm{K} \mathrm{km} \mathrm{s}^{-1} \mathrm{pc}^{2}\right)\end{array}$ & $\begin{array}{l}\alpha_{\mathrm{CO}}^{(* *)} \\
\left(M_{\odot}\left(\mathrm{K} \mathrm{km} \mathrm{s}^{-1} \mathrm{pc}^{2}\right)^{-1}\right)\end{array}$ \\
\hline North & $1.29 \pm 0.07$ & $437.3 \pm 26.1$ & $67.5 \pm 6.3$ & $6.5 \pm 0.7$ \\
South & $1.04 \pm 0.04$ & $732.9 \pm 37.0$ & $47.7 \pm 4.5$ & $15.3 \pm 1.6$ \\
\hline
\end{tabular}

Notes. ${ }^{(*)}$ Corrected for spatial broadening. ${ }^{(*)}$ Assumes a $\mathrm{CO}(2-1)$ to $\mathrm{CO}(1-0)$ ratio $r_{21} \sim 1$.

$\mathrm{CO}(2-1)$ integrated flux density in $\mathrm{Jy} \mathrm{km} \mathrm{s}^{-1}$, obtained from the ALMA and APEX combined $\mathrm{CO}(2-1)$ line cube, and $D$ is the distance to Magellanic Bridge in Mpc. The resulting luminosities are $L_{\mathrm{CO}(2-1)}=67.5 \pm 6.3 \mathrm{~K} \mathrm{~km} \mathrm{~s}^{-1} \mathrm{pc}^{2}$ for $\mathrm{CO}$ cloud North, and $L_{\mathrm{CO}(2-1)}=47.7 \pm 4.5 \mathrm{~K} \mathrm{~km} \mathrm{~s}^{-1} \mathrm{pc}^{2}$ for $\mathrm{CO}$ cloud South.

We compared the Magellanic Bridge A total luminosity measured by the single-dish APEX $\mathrm{CO}(2-1)$ observations and by the ALMA $\mathrm{CO}(2-1)$ observations to determine any missing diffuse emission. The total luminosity detected by APEX, measured inside a a $58^{\prime \prime} \times 58^{\prime \prime}$ box centered at $\alpha=1^{\mathrm{h}} 43^{\mathrm{m}} 51^{\mathrm{s}} .3$, $\delta=-74^{\circ} 32^{\prime} 09^{\prime} .2$, is $134 \pm 9 \mathrm{~K} \mathrm{~km} \mathrm{~s}^{-1} \mathrm{pc}^{2}$. The luminosity of clouds North and South in the ALMA CO(2-1) line cube is $66.7 \pm 6.0$ and $48.4 \pm 4.2 \mathrm{~K} \mathrm{~km} \mathrm{~s}^{-1} \mathrm{pc}^{2}$, respectively. If we add the CO luminosities of the two clouds, we obtain a total luminosity for Magellanic Bridge A of $115 \pm 10 \mathrm{~K} \mathrm{~km} \mathrm{~s}^{-1} \mathrm{pc}^{2}$. Thus, ALMA recovers, within the uncertainties, almost all the luminosity measured in the APEX $\mathrm{CO}(2-1)$ line cube, implying that most of the emission in Magellanic Bridge A is concentrated in the two $\mathrm{CO}(2-1)$ clouds we characterize in this work.

We determined the virial masses $M_{\text {vir }}$ of the clouds using Eq. (3) of MacLaren et al. (1988),

$M_{\mathrm{vir}}=190 R(\Delta v)^{2}$,

where $R$ is the radius in pc, $\Delta v$ is the FWHM of the line emission in $\mathrm{km} \mathrm{s}^{-1}$, and $M_{\mathrm{vir}}$ is in $M_{\odot}$. This equation assumes sphericalshaped gravitationally bound molecular clouds and a density profile $\rho \propto r^{-1}$. We used the deconvolved radii in Table 7 and the FWHM in Table 6. The virial mass for CO source North is $437.3 \pm 26.1 M_{\odot}$ and for source South it is $732.9 \pm 37.0$ $M_{\odot}$. Even though these clouds are parsec-sized, they contain $\sim 5 \times 10^{2} M_{\odot}$, similar to the masses of the most massive molecular clumps in the Milky Way, which have typical radii of $\sim 1 \mathrm{pc}$ (see Bergin \& Tafalla 2007, and references within).

We estimated the CO-to- $\mathrm{H}_{2}$ conversion factor $\alpha_{\mathrm{CO}}=$ $M_{\mathrm{vir}} / L_{\mathrm{CO}}$ and the corresponding $X_{\mathrm{CO}}$, which is usually derived for the ${ }^{12} \mathrm{CO}(1-0)$ emission. We did not attempt to correct for variations in the $J=2-1$ to $J=1-0$ ratio $r_{21}$, and simply assumed $r_{21} \sim 1$ for the purposes of comparison, which is also a common result for SMC observations (e.g., Lequeux et al. 1994; Rubio et al. 1996). For CO cloud North the derived conversion factor is $\alpha_{\mathrm{CO}}=6.5 \pm 0.7 M_{\odot}\left(\mathrm{K} \mathrm{kms}^{-1} \mathrm{pc}^{2}\right)^{-1}$ $\left(X_{C O}=(3.0 \pm 0.3) \times 10^{20} \mathrm{~cm}^{-2}\left(\mathrm{~K} \mathrm{~km} \mathrm{~s}^{-1}\right)^{-1}\right)$ and for cloud South $\alpha_{\mathrm{CO}}=15.3 \pm 1.6 \quad M_{\odot} \quad\left(\mathrm{K} \quad \mathrm{km} \quad \mathrm{s}^{-1} \mathrm{pc}^{2}\right)^{-1}$ $\left(X_{\mathrm{CO}}=(7.1 \pm 0.7) \times 10^{20} \mathrm{~cm}^{-2}\left(\mathrm{~K} \mathrm{~km} \mathrm{~s}^{-1}\right)^{-1}\right)$. These values are 1.5 and 3.6 times the canonical value for the Milky Way $\alpha_{\mathrm{CO}}(M W)=4.3 M_{\odot}\left(\mathrm{K} \mathrm{km} \mathrm{s}^{-1} \mathrm{pc}^{2}\right)^{-1}\left(X_{\mathrm{CO}}=2 \times\right.$ $\left.10^{20} \mathrm{~cm}^{-2}(\mathrm{~K} \mathrm{~km} / \mathrm{s})^{-1}\right)$.

The derived properties $R, M_{\mathrm{vir}}, L_{\mathrm{CO}(2-1)}$ and $\alpha_{\mathrm{CO}}$ are summarized in Table 7.

\section{Discussion}

In this section we discuss our results and compare them to previous observations of Magellanic Bridge A and other lowmetallicity galaxies.

\section{1. $\mathrm{CO}(2-1)$ in comparison with previous studies}

The molecular clouds we found in $\mathrm{CO}(2-1)$ emission have spectral properties and masses similar to those inferred from previous unresolved $\mathrm{CO}$ measurements in Magellanic Bridge A. The central velocities of CO sources North and South, $174.6 \pm 0.6$ and $174.0 \pm 0.9 \mathrm{~km} \mathrm{~s}^{-1}$, respectively, are close to the $174.7 \mathrm{~km} \mathrm{~s}^{-1}$ reported for Magellanic Bridge A by Mizuno et al. (2006) through ${ }^{12} \mathrm{CO}(1-0)$ emission with NANTEN (beam $F W H M=2.6^{\prime}$ ). The linewidths of the CO clouds, $1.33 \pm 0.03 \mathrm{~km} \mathrm{~s}^{-1}$ for cloud North and $1.93 \pm 0.05 \mathrm{~km} \mathrm{~s}^{-1}$ for cloud South, are consistent with the ${ }^{12} \mathrm{CO}(1-0)$ velocity FWHM of $1.6 \mathrm{~km} \mathrm{~s}^{-1}$ reported for Magellanic Bridge A in Mizuno et al. (2006) and with the ${ }^{12} \mathrm{CO}(3-2)$ FWHM of $1.4 \mathrm{~km} \mathrm{~s}^{-1}$, reported in Muller et al. (2014) observed with ASTE Telescope $\left(F W H M=22^{\prime \prime}\right)$. The sum of the virial masses of the $\mathrm{CO}$ clouds North and South, $(1.17 \pm 0.06) \times 10^{3} M_{\odot}$ is in agreement with the mass estimated for Magellanic Bridge A by Mizuno et al. (2006), $10^{3} M_{\odot}$, using $I_{\mathrm{CO}(1-0)}$ and a conversion factor of $X_{\mathrm{CO}}=1.4 \times 10^{21} \mathrm{~cm}^{-2}\left(\mathrm{~K} \mathrm{~km} \mathrm{~s}^{-1}\right)^{-1}$.

Comparing the $\mathrm{CO}$ luminosities found by previous studies of this source is not easy as they measure different rotational transitions with different resolutions and sensitivities. Nevertheless, by comparing the luminosities over matched areas, together with the similarities in velocity FWHM and virial mass, we 
estimate that the emission detected in previous observations is mainly associated with the two clouds we observed in this work. The ${ }^{12} \mathrm{CO}(1-0)$ luminosity in the NANTEN detection of Magellanic Bridge $\mathrm{A}$ is $L_{\mathrm{CO}(1-0)}=70 \pm 8 \mathrm{~K} \mathrm{~km} \mathrm{~s}^{-1} \mathrm{pc}^{2}$ (Mizuno et al. 2006). The ${ }^{12} \mathrm{CO}(3-2)$ luminosity in the ASTE detection is $L_{\mathrm{CO}(3-2)}=64 \pm 8 \mathrm{~K} \mathrm{~km} \mathrm{~s}^{-1} \mathrm{pc}^{2}$ (Muller et al. 2014). The total $\mathrm{CO}(2-1)$ luminosity we measured from both Magellanic Bridge A clouds is $L_{\mathrm{CO}(2-1)}=115 \pm 11 \mathrm{~K} \mathrm{~km} \mathrm{~s}^{-1} \mathrm{pc}^{2}$. The resulting ${ }^{12} \mathrm{CO}(3-2) /(2-1)$ luminosity ratio is $r_{32}=0.56 \pm 0.09$, which is reasonable for a star-forming cloud. The ${ }^{12} \mathrm{CO}(2-1) /(1-0)$ ratio is $r_{21}=1.64 \pm 0.24$, which is unusually high with respect to typical values observed in the Milky Way, the Magellanic Clouds, and other galaxies where $r_{21} \sim 0.7-1.1$ (e.g., Rubio et al. 1996; Sorai et al. 2001; Bolatto et al. 2003; Nikolić et al. 2007). Although in localized regions higher ratios can be observed, caused by effective optical depth (Bolatto et al. 2003), it is also possible that the NANTEN ${ }^{12} \mathrm{CO}(1-0)$ luminosity is underestimated. Because of the similarities in velocity, FWHM, and luminosity, it seems that most (if not all) of the $\mathrm{CO}$ emission from Magellanic Bridge A detected in previous studies comes from the two compact clouds presented in this work.

The CO emission from the North and South clouds show interesting differences from parsec-sized $\mathrm{CO}$ sources present in the Milky Way. Their virial masses are one order of magnitude higher, implying higher densities in the gas. At radii between 1 and $2 \mathrm{pc}$, clouds in the Milky Way have masses between a few $10 M_{\odot}$ to a few $100 M_{\odot}$ (Miville-Deschênes et al. 2017), while the clouds found in Magellanic Bridge A have $400 M_{\odot}$ and 700 $M_{\odot}$ for North and South, respectively. We note, however, that most of the clouds in the Miville-Deschênes et al. (2017) catalog have large virial parameters, and as a consequence are not self-gravitating and are unlikely to be forming stars. The assumption of virial or self-gravitating equilibrium in the Magellanic Bridge A clouds may not be strictly correct, but it is not unreasonable for star-forming clouds. Clouds that have an excess of kinetic energy with respect to their potential energy will be short-lived and very unlikely to locally collapse to form stars. If the CO-emitting cloud is embedded in a large envelope, part of the velocity dispersion may be associated with the external confining pressure associated with the weight of the envelope. In that case the virial mass estimate effectively includes part of the mass of the envelope. Using the virial mass estimate, the $\mathrm{CO}$ clouds North and South have surface densities $\Sigma_{\mathrm{mol}} \approx 80$ and $230 M_{\odot} \mathrm{pc}^{-2}$, respectively, and corresponding bulk densities $n\left(\mathrm{H}_{2}\right) \sim 700$ and $2600 \mathrm{~cm}^{-3}$ assuming spherical geometry. The surface densities of Magellanic Bridge A clouds are similar to typical $\Sigma_{\text {mol }}$ for star-forming clouds in the Milky Way disk (e.g., Heiderman et al. 2010; Evans et al. 2014), but their bulk volume densities are higher than commonly found bulk densities in our Galaxy, which is a reflection of the small size of the clouds in this work. Roman-Duval et al. (2010) find $\Sigma_{\text {mol }} \sim 144 M_{\odot} \mathrm{pc}^{-2}$ and $n\left(\mathrm{H}_{2}\right) \sim 230 \mathrm{~cm}^{-3}$ as an average for Milky Way clouds in the Galactic Ring Survey, which tend to be star-forming clouds. The Magellanic Bridge clouds have volume densities that are similar to the densities of clumps in local star-forming molecular clouds (e.g., Evans et al. 2014). This supports the idea that what we see emitting brightly in $\mathrm{CO}$ at low metallicities corresponds to the denser regions of molecular clouds.

In other aspects, the Magellanic Bridge A clouds are similar to clouds found in the Magellanic System and other low-metallicity galaxies. Their $\mathrm{CO}(2-1)$ emission has similar velocity dispersion and luminosity to other $\mathrm{CO}$ clouds studied in the Magellanic Bridge. Molecular clouds with radii $0.3-1 \mathrm{pc}$, velocity FWHM $1-2 \mathrm{~km} \mathrm{~s}^{-1}$ and CO luminosities
$10-100 \mathrm{~K} \mathrm{~km} \mathrm{~s}^{-1} \mathrm{pc}^{2}$ have been found in Magellanic Bridge B (Saldaño et al. 2018). Magellanic Bridge $\mathrm{C}^{12} \mathrm{CO}(1-0)$ emission is concentrated in clouds with radii $0.9-1.5 \mathrm{pc}$ and velocity FWHM $0.5-1.4 \mathrm{~km} \mathrm{~s}^{-1}$ (Kalari et al. 2020), similar to the clouds we found in this work. The Magellanic Bridge A clouds have similar radii and slightly narrower linewidths and luminosities than regions in the LMC and SMC with active star formation, observed at similar (subparsec) resolution. For example, in 30 Doradus in the LMC, clouds with radii $\gtrsim 0.5 \mathrm{pc}$ show lines $1-6 \mathrm{~km} \mathrm{~s}^{-1}$ wide and $\mathrm{CO}(2-1)$ luminosities $\gtrsim 100 \mathrm{~K} \mathrm{~km} \mathrm{~s}^{-1} \mathrm{pc}^{2}$ (Indebetouw et al. 2013). In N83 in the SMC, clouds with radii $\sim 0.8 \mathrm{pc}$ have linewidths of $\sim 4 \mathrm{~km} \mathrm{~s}^{-1}$ and $\mathrm{CO}(2-1)$ luminosities $100-200 \mathrm{~K} \mathrm{~km} \mathrm{~s}^{-1} \mathrm{pc}^{2}$ (Muraoka et al. 2017). The narrower linewidths in Magellanic Bridge A may be the consequence of lower cloud masses, or alternatively lower external pressures (Field et al. 2011). Small clouds (1-3 pc radii) with linewidths around $2-3 \mathrm{~km} \mathrm{~s}^{-1}$ and high densities $\left(n\left(\mathrm{H}_{2}\right) \sim 10^{3} \mathrm{~cm}^{-3}\right)$ have been reported in dwarf galaxies like WLM (Rubio et al. 2015) and NGC6822 (Schruba et al. 2017). In general, our results support the idea that $\mathrm{CO}$ in low-metallicity galaxies traces dense regions of the molecular clouds, and is detected as compact dense $\mathrm{CO}$ clouds with narrow linewidths (Rubio et al. 2015).

The $\alpha_{\mathrm{CO}}$ CO-to- $\mathrm{H}_{2}$ conversion factor values estimated in this work using the virial masses are around 2-4 times larger than the standard Milky Way disk value $\alpha_{\mathrm{CO}}(M W)=$ $4.3 M_{\odot}\left(\mathrm{K} \mathrm{kms}^{-1} \mathrm{pc}^{2}\right)^{-1}$. Although large conversion factors are observed on large scales for low-metallicity galaxies, observations on small scales and in regions where $\mathrm{CO}$ is bright in low-metallicity cloud complexes frequently yield conversion factors $\alpha_{\mathrm{CO}}$ similar to the canonical value for the Galaxy (e.g., Rubio et al. 2015; Schruba et al. 2017; Wong et al. 2017; Jameson et al. 2018). Rubio et al. (1993) found that in the SMC, $X_{\mathrm{CO}}$ decreases with cloud size as $\log X_{\mathrm{SMC}}=0.7 \log R+20.26$ between 10 and $100 \mathrm{pc}$. Extending their relation for cloud sizes $R \sim 1 \mathrm{pc}$, the resulting value of $X_{\mathrm{CO}}$ is almost the canonical galactic value. For $\mathrm{CO}$ cloud North, $\alpha_{\mathrm{CO}}$ is in good agreement with the value obtained for clouds sizes of $\sim 2 \mathrm{pc}$ using the Rubio et al. (1993) relation. Therefore, the $\alpha_{\mathrm{CO}}$ values found in this work are consistent with $\alpha_{\mathrm{CO}}$ values found for other low-metallicity regions at similar resolution.

\subsection{Gas and dust comparison}

We compared the molecular gas and dust emission in Magellanic Bridge A by convolving the $\mathrm{CO}(2-1)$ ALMA and APEX combined cube to $22^{\prime \prime}$ resolution. We integrated the convolved $\mathrm{CO}(2-1)$ emission between 172 and $176 \mathrm{~km} \mathrm{~s}^{-1}$. The rms noise of the resultant image is $\sigma=1.8 \mathrm{Jy}_{\text {beam }}^{-1} \mathrm{~km} \mathrm{~s}^{-1}$. The $\operatorname{CO}(2-1)$ peaks can still be distinguished as separate sources at this resolution, but they share the same $3 \sigma$ and $5 \sigma$ contours (see Fig. 6).

At this common resolution we can characterize the relative sizes of the sources in dust and $\mathrm{CO}$ by fitting twodimensional Gaussians. In $\mathrm{CO}(2-1)$, the FWHM of the Gaussians are $(7.1 \pm 0.2) \mathrm{pc} \times(6.6 \pm 0.2) \mathrm{pc}$ for cloud North, and $(5.6 \pm 0.4) \mathrm{pc} \times(4.9 \pm 0.3) \mathrm{pc}$ for cloud South. Sources North and South in the $870 \mu \mathrm{m}$ continuum are described by Gaussians with FWHM of $(10.3 \pm 0.6) \mathrm{pc} \times(10.0 \pm 0.7) \mathrm{pc}$ and $(16.9 \pm 1.1) \mathrm{pc} \times(16.5 \pm 0.5) \mathrm{pc}$, respectively. The ratio of the continuum to $\mathrm{CO}$ spatial size for the cloud North source is $1.5 \pm 0.1$, while for cloud South it is $3.0 \pm 0.3$. This difference in size (in particular for cloud South) suggests the presence of an extended halo of cold material, possibly molecular, that is 


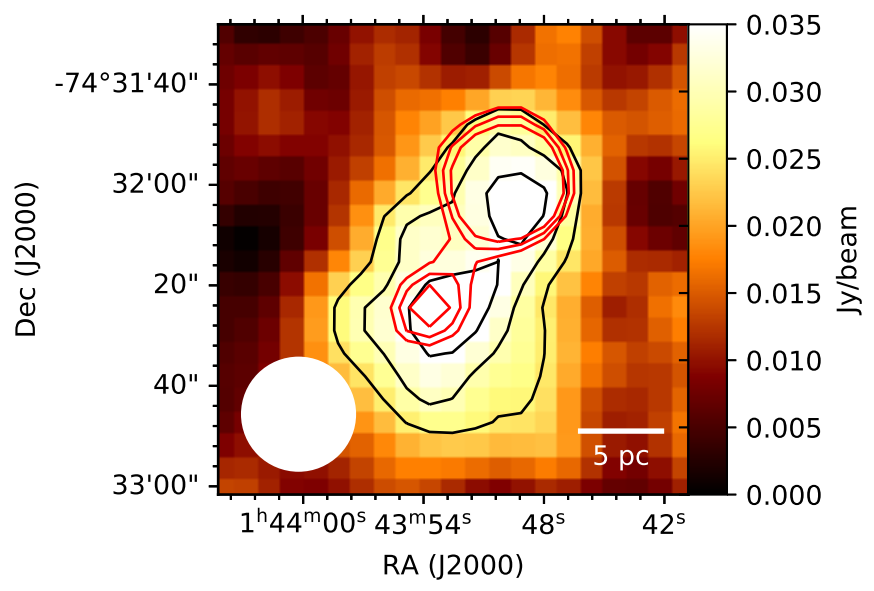

Fig. 6. LABOCA continuum image at $870 \mu \mathrm{m}$, with black contours placed at 25, 30, and $35 \mathrm{mJy}^{-1} \mathrm{bem}^{-1}$, as in Fig. 3. The white circle represents the beam size (beam $F W H M=22^{\prime \prime}$ ). Red contours correspond to the ALMA and APEX $\mathrm{CO}(2-1)$ combined line emission convolved to the APEX resolution of $22^{\prime \prime}$, integrated between 172 and $176 \mathrm{~km} \mathrm{~s}^{-1}$, at $5 \sigma, 6 \sigma$, and $7 \sigma$, where $\sigma$ is the rms of the integrated image $\left(\sigma=1.8 \mathrm{Jy}_{\text {beam }}^{-1} \mathrm{~km} \mathrm{~s}^{-1}\right)$. The scalebar in the lower right corner represents a 5 pc length.

not bright in $\mathrm{CO}$. The expectation from photodissociation region models is that at low metallicity the region of a cloud that emits brightly in $\mathrm{CO}$ shrinks due to the diminished extinction caused by the lower dust-to-gas ratio (Bolatto et al. 2013).

It is interesting to note that not only is the size of the emission larger in the long-wavelength dust continuum, but the gas mass estimates from the FIR dust continuum MBB fit to sources North and South are also a factor of $\sim 3-4$ higher than virial masses from $\mathrm{CO}$ (see Tables 4 and 7). This result is along the lines of previous studies in the SMC, where the total gas mass derived using $\mathrm{CO}$ emission is substantially lower than the cold gas mass obtained from modeling the dust emission (Rubio et al. 2004; Leroy et al. 2007; Bot et al. 2007, 2010; Bolatto et al. 2011; Jameson et al. 2016). It is consistent with the idea that the CO-emitting regions of molecular clouds at low metallicity are encapsulated in a much larger cold gas envelope, likely molecular, that is not emitting in CO.

\subsection{Gas and dust in the context of star formation}

The North and South clouds in Magellanic Bridge A show differences in the mid-infrared. In the 8 and $24 \mu \mathrm{m}$ emission images of SAGE-SMC program, source North is detected with a flux of $0.9 \pm 0.1$ and $1.0 \pm 0.1 \mathrm{mJy}$, respectively, whereas source South has a flux density of $7.1 \pm 0.3$ and $60.0 \pm 0.7 \mathrm{mJy}$ (Gordon et al. 2011). The $8 \mu \mathrm{m}$ emission is associated with the presence of polycyclic aromatic hydrocarbons (PAHs), which are components of dust and are usually correlated with star formation activity (Chen et al. 2014). The $24 \mu \mathrm{m}$ emission is a tracer of warm dust, which is associated with massive star formation. The difference in fluxes at $24 \mu \mathrm{m}$ is consistent with the presence of a B-type star towards the South cloud.

The $\mathrm{CO}(2-1)$ clouds in Magellanic Bridge A have ongoing star formation, with associated YSOs. The North source coincides with a faint YSO candidate (J014349.20-743200.63) classified by Chen et al. (2014), while the South source coincides with a brighter YSO (J014353.94-743224.71) classified as an embedded source in the same work. The South source hosts a compact multiple system, where a B-type star dominates the far-ultraviolet (FUV) light, while the YSO in this location dominates the near-infrared and mid-infrared light (Chen et al. 2014). The dust temperature reflects this difference in stellar content: the South cloud is being heated by the multiple stars it hosts, in particular a B-type star, while the North cloud might be mostly heated by its fainter YSO. Therefore, it seems that the South molecular cloud has already had an episode of massive star formation, and indeed Chen et al. (2014) speculate that the formation of a group of early B stars 5-10 Myr ago and the corresponding expansion of their H II regions (still visible in $\mathrm{H} \alpha$ ) may have triggered the current star formation activity in Magellanic Bridge A.

\section{Summary and conclusions}

We characterized the molecular gas and dust emission from the Magellanic Bridge A molecular clouds using ALMA $1.3 \mathrm{~mm}$ continuum and $\mathrm{CO}(2-1)$ observations at subparsec resolution, together with APEX $870 \mu$ m continuum and $\mathrm{CO}(2-1)$ line observations at $\sim 6 \mathrm{pc}$ resolution and Spitzer and Herschel FIR archival data.

At this resolution, Magellanic Bridge A separates into two components, North and South. Their dust emission has temperatures of $T_{\mathrm{d}}=21.7 \pm 2.2 \mathrm{~K}$ and $T_{\mathrm{d}}=24.5 \pm 1.1 \mathrm{~K}$, respectively, with an emissivity exponent $\beta \approx 1.4$ obtained from the MBB fitting to the FIR emission of Magellanic Bridge A at $\lambda \leq 500 \mu \mathrm{m}$. The difference in temperature is consistent with the fact that the North source is not detected at 100 and $160 \mu \mathrm{m}$, and that its star formation activity seems to be weaker than that of the South source. Using the MBB model that best fits the FIR dust fluxes, we obtained total gas masses of $(1.3 \pm 0.3) \times 10^{3} M_{\odot}$ at source North and $(2.9 \pm 1.2) \times 10^{3} M_{\odot}$ at source South, and a total gas mass of $(6.8 \pm 1.5) \times 10^{3} M_{\odot}$ for the entire complex.

After removing possible contributions from free-free and $\mathrm{CO}$ line emission, the bolometer measurement at $870 \mu \mathrm{m}$ from LABOCA shows a very significant submillimeter excess of a factor of $\sim 6.7$ for source North and 3.0 for source South over their FIR fits. The $870 \mu \mathrm{m}$ image exhibits morphology consistent with that of the FIR images, peaking at the location of the $\mathrm{CO}$ clouds. The calibration of the data does not appear to be at fault, and the noise in the image is entirely consistent with a priori expectations: without the excess there would have been a marginal detection of the source. A similar excess, however, is not detected in the ALMA $12 \mathrm{~m}$ and Morita Array imaging at $1.3 \mathrm{~mm}$. The upper limits for flux density at $1.3 \mathrm{~mm}$ are consistent with the predicted fluxes by the MBB model. This requires that either emission is extended enough that it is filtered out by the interferometer or that the excess is caused by a process that peaks at $870 \mu \mathrm{m}$ (perhaps similar to the Anomalous Microwave Emission detected at lower frequencies; Dickinson et al. 2018). High-resolution observations at $870 \mu \mathrm{m}$ with ALMA will help to establish the nature of the excess.

We found dense molecular clouds in Magellanic Bridge A using ALMA+APEX combined $\mathrm{CO}(2-1)$ line emission, which spatially coincide with the North and South dust sources. These two clouds have radii $\sim 1 \mathrm{pc}$, velocity FWHM of 1.3 and $1.9 \mathrm{~km} \mathrm{~s}^{-1}$ and virial masses of $\sim 400$ and $700 M_{\odot}$ for clouds North and South, respectively. Accordingly, their bulk volume densities are $n\left(\mathrm{H}_{2}\right) \sim 700$ and $2600 \mathrm{~cm}^{-3}$, significantly higher than the typical density of clouds in the Milky Way $\left(n \sim 230 \mathrm{~cm}^{-3}\right.$; Roman-Duval et al. 2010). The total interferometric $L_{\mathrm{CO}(2-1)}$ of the complex is $85 \%$ of the luminosity $L_{\mathrm{CO}(2-1)}$ measured with the APEX single dish, which suggests that most (if not all) $\mathrm{CO}(2-1)$ emission from Magellanic Bridge 
A comes from these two clouds. Using the virial mass, we find a CO-to- $\mathrm{H}_{2}$ conversion factor $\alpha_{\mathrm{CO}}$ of $6.5 \pm 1.2 M_{\odot}\left(\mathrm{K} \mathrm{kms}^{-1}\right.$ $\left.\mathrm{pc}^{2}\right)^{-1}$ and $15.3 \pm 1.6 M_{\odot}\left(\mathrm{K} \mathrm{km} \mathrm{s} \mathrm{pc}^{-1}\right)^{-1}$ for the North and South clouds respectively, consistent with the trend of $\alpha_{\mathrm{CO}}$ approaching Milky Way values on the small spatial scales.

We compared the $\mathrm{CO}(2-1)$ and dust emissions at a common resolution of $22^{\prime \prime}$. We find that $\mathrm{CO}(2-1)$ emission covers a smaller area than continuum emission in the South cloud, whereas they have a similar spatial extent on the more quiescent North cloud. The total gas mass derived from the dust emission is $\sim 4$ times higher than the sum of virial masses obtained for both clouds.

Acknowledgements. We thank the anonymous referee for very constructive feedback and comments which helped to improve the quality of this article. M.T.V acknowledges financial support from CONICYT (Chile) through the scholarship CONICYT-PFCHA/ Magister Nacional 2018 - 22180279, Universidad de Chile VID grant ENL22/18 and FONDECYT grant No1190684. M.R. wishes to acknowledge support from Universidad de Chile VID grant ENL22/18, from CONICYT (CHILE) through FONDECYT grant No1190684, and partial support from CONICYT project Basal AFB-170002. H.P.S acknowledges financial support from a fellowship from Consejo Nacional de Investigaciones Científicas y Técnicas (CONICET), and from Secretaría de Ciencias y Técnicas (Secyt), Córdoba, Argentina. This paper makes use of the following ALMA data: ADS/JAO.ALMA\#2012.1.00683.S. ALMA is a partnership of ESO (representing its member states), NSF (USA) and NINS (Japan), together with NRC (Canada), MOST and ASIAA (Taiwan), and KASI (Republic of Korea), in cooperation with the Republic of Chile. The Joint ALMA Observatory is operated by ESO, AUI/NRAO and NAOJ. This publication is based on data acquired with the Atacama Pathfinder Experiment (APEX). This research made use of APLpy, an open-source plotting package for Python (Robitaille \& Bressert 2012). This research made use of Astropy (http: //wWw . astropy .org), a communitydeveloped core Python package for Astronomy (Astropy Collaboration 2013 , 2018).

\section{References}

Astropy Collaboration (Robitaille, T. P., et al.) 2013, A\&A, 558, A33 Astropy Collaboration (Price-Whelan, A. M., et al.) 2018, AJ, 156, 123 Bendo, G. J., Dale, D. A., Draine, B. T., et al. 2006, ApJ, 652, 283 Bergin, E. A., \& Tafalla, M. 2007, ARA\&A, 45, 339

Besla, G., Kallivayalil, N., Hernquist, L., et al. 2012, MNRAS, 421, 2109

Bica, E. L. D., \& Schmitt, H. R. 1995, ApJS, 101, 41

Bica, E., Santiago, B., Bonatto, C., et al. 2015, MNRAS, 453, 3190

Bolatto, A. D., Leroy, A., Israel, F. P., \& Jackson, J. M. 2003, ApJ, 595, 167

Bolatto, A. D., Leroy, A. K., Jameson, K., et al. 2011, ApJ, 741, 12

Bolatto, A. D., Wolfire, M., \& Leroy, A. K. 2013, ARA\&A, 51, 207

Bot, C., Boulanger, F., Rubio, M., \& Rantakyro, F. 2007, A\&A, 471, 103

Bot, C., Rubio, M., Boulanger, F., et al. 2010, A\&A, 524, A52

Chen, C. H. R., Indebetouw, R., Muller, E., et al. 2014, ApJ, 785, 162

Cioni, M. R. L., van der Marel, R. P., Loup, C., \& Habing, H. J. 2000, A\&A, 359, 601

Dickinson, C., Ali-Haïmoud, Y., Barr, A., et al. 2018, New Astron. Rev., 80, 1

Drabek, E., Hatchell, J., Friberg, P., et al. 2012, MNRAS, 426, 23

Evans, Neal J. I., Heiderman, A., \& Vutisalchavakul, N. 2014, ApJ, 782, 114

Field, G. B., Blackman, E. G., \& Keto, E. R. 2011, MNRAS, 416, 710

Galametz, M., Madden, S., Galliano, F., et al. 2009, A\&A, 508, 645

Galametz, M., Albrecht, M., Kennicutt, R., et al. 2014, MNRAS, 439, 2542

Galliano, F., Madden, S. C., Jones, A. P., et al. 2003, A\&A, 407, 159

Galliano, F., Madden, S. C., Jones, A. P., Wilson, C. D., \& Bernard, J. P. 2005, A\&A, 434, 867

Gardiner, L. T., Sawa, T., \& Fujimoto, M. 1994, MNRAS, 266, 567

Gaustad, J. E., McCullough, P. R., Rosing, W., \& Van Buren, D. 2001, PASP, 113,1326
Gordon, K. D., Bot, C., Muller, E., et al. 2009, ApJ, 690, L76

Gordon, K. D., Meixner, M., Meade, M. R., et al. 2011, AJ, 142, 102

Gordon, K. D., Roman-Duval, J., Bot, C., et al. 2014, ApJ, 797, 85

Harries, T. J., Hilditch, R. W., \& Howarth, I. D. 2003, MNRAS, 339, 157

Harris, J. 2007, ApJ, 658, 345

Heiderman, A., Evans, Neal J. I., Allen, L. E., Huard, T., \& Heyer, M. 2010, ApJ, 723, 1019

Hermelo, I., Relaño, M., Lisenfeld, U., et al. 2016, A\&A, 590, A56

Hildebrand, R. H. 1983, QJRAS, 24, 267

Hindman, J. V., Kerr, F. J., \& X.McGee, R. 1963, Aust. J. Phys., 16, 570

Hunt, L. K., Dyer, K. K., Thuan, T. X., \& Ulvestad, J. S. 2004, ApJ, 606 853

Indebetouw, R., Brogan, C., Chen, C. H. R., et al. 2013, ApJ, 774, 73

Jameson, K. E., Bolatto, A. D., Leroy, A. K., et al. 2016, ApJ, 825, 12

Jameson, K. E., Bolatto, A. D., Wolfire, M., et al. 2018, ApJ, 853, 111

Kalari, V. M., Carraro, G., Evans, C. J., \& Rubio, M. 2018, ApJ, 857, 132

Kalari, V. M., Rubio, M., Saldaño, H. P., \& Bolatto, A. D. 2020 MNRAS, submitted

Lee, J. K., Rolleston, W. R. J., Dufton, P. L., \& Ryans, R. S. I. 2005, A\&A, 429, 1025

Lehner, N., Sembach, K. R., Dufton, P. L., Rolleston, W. R. J., \& Keenan, F. P. 2001, ApJ, 551, 781

Lehner, N., Howk, J. C., Keenan, F. P., \& Smoker, J. V. 2008, ApJ, 678, 219

Lequeux, J., Le Bourlot, J., Pineau des Forets, G., et al. 1994, A\&A, 292, 371

Leroy, A., Bolatto, A., Stanimirovic, S., et al. 2007, ApJ, 658, 1027

MacLaren, I., Richardson, K. M., \& Wolfendale, A. W. 1988, ApJ, 333, 821

McClure-Griffiths, N. M., Pisano, D. J., Calabretta, M. R., et al. 2009, ApJS, 181, 398

McMullin, J. P., Waters, B., Schiebel, D., Young, W., \& Golap, K. 2007, in ASP Conf. Ser., 376, 127

Meixner, M., Panuzzo, P., Roman-Duval, J., et al. 2013, AJ, 146, 62

Miville-Deschênes, M.-A., Murray, N., \& Lee, E. J. 2017, ApJ, 834, 57

Mizuno, A., Onishi, T., Yonekura, Y., et al. 1995, ApJ, 445, L161

Mizuno, A., Yamaguchi, R., Tachihara, K., et al. 2001, PASJ, 53, 1071

Mizuno, N., Muller, E., Maeda, H., et al. 2006, ApJ, 2, 107

Muller, E., \& Parker, Q. A. 2007, PASA, 24, 69

Muller, E., Staveley-Smith, L., Zealey, W., \& Stanimirovic, S. 2003a, MNRAS, 339,105

Muller, E., Staveley-Smith, L., \& Zealey, W. J. 2003b, MNRAS, 338, 609

Muller, E., Mizuno, N., Minamidani, T., et al. 2014, PASJ, 66, 4

Murai, T., \& Fujimoto, M. 1980, PASJ, 32, 581

Muraoka, K., Homma, A., Onishi, T., et al. 2017, ApJ, 844, 98

Nikolić, S., Garay, G., Rubio, M., \& Johansson, L. E. B. 2007, A\&A, 471, 561

Robitaille, T., \& Bressert, E. 2012, APLpy: Astronomical Plotting Library in Python

Rolleston, W. R. J., Venn, K., Tolstoy, E., \& Dufton, P. L. 2003, A\&A, 400, 21

Roman-Duval, J., Jackson, J. M., Heyer, M., Rathborne, J., \& Simon, R. 2010, ApJ, 723, 492

Rosolowsky, E., \& Leroy, A. 2006, PASP, 118, 590

Rubio, M., Lequeux, J., \& Boulanger, F. 1993, A\&A, 271, 9

Rubio, M., Lequeux, J., Boulanger, F., et al. 1996, A\&AS, 118, 263

Rubio, M., Boulanger, F., Rantakyro, F., \& Contursi, A. 2004, A\&A, 425, L1

Rubio, M., Elmegreen, B. G., Hunter, D. A., et al. 2015, Nature, 525, 218

Saldaño, H. P., Rubio, M., Jameson, K., \& Bolatto, A. D. 2018, Bol. Asoc. Argent. Astron. Plata Argent., 60, 192

Schlafly, E. F., \& Finkbeiner, D. P. 2011, ApJ, 737, 103

Schruba, A., Leroy, A. K., Kruijssen, J. M. D., et al. 2017, ApJ, 835, 278

Shetty, R., Kauffmann, J., Schnee, S., \& Goodman, A. A. 2009, ApJ, 696, 676

Siringo, G., Kreysa, E., Kovács, A., et al. 2009, A\&A, 497, 945

Sorai, K., Hasegawa, T., Booth, R. S., et al. 2001, ApJ, 551, 794

Staveley-Smith, L., Kim, S., Putman, M., \& Stanimirovic, S. 1998, RvMA, 11, 117

Vassilev, V., Meledin, D., Lapkin, I., \& Belitsky, V. 2008, A\&A, 490, 1157

Verdugo, C. 2012, Master's thesis, Universidad de Chile, Chile

Virtanen, P., Gommers, R., Oliphant, T. E., et al. 2020, Nat. Methods, 17, 261

Wong, T., Hughes, A., Tokuda, K., et al. 2017, ApJ, 850, 139 